\title{
Transition of unsteady velocity profiles with reverse flow
}

\author{
By DEBOPAM DAS AND JAYWANT H. ARAKERI \\ Department of Mechanical Engineering, Indian Institute of Science, Bangalore-560 012, India \\ e-mail: debopam@mecheng.iisc.ernet.in; jaywant@mecheng.iisc.ernet.in
}

(Received 5 August 1996 and in revised form 15 June 1998)

This paper deals with the stability and transition to turbulence of wall-bounded unsteady velocity profiles with reverse flow. Such flows occur, for example, during unsteady boundary layer separation and in oscillating pipe flow. The main focus is on results from experiments in time-developing flow in a long pipe, which is decelerated rapidly. The flow is generated by the controlled motion of a piston. We obtain analytical solutions for laminar flow in the pipe and in a two-dimensional channel for arbitrary piston motions. By changing the piston speed and the length of piston travel we cover a range of values of Reynolds number and boundary layer thickness. The velocity profiles during the decay of the flow are unsteady with reverse flow near the wall, and are highly unstable due to their inflectional nature. In the pipe, we observe from flow visualization that the flow becomes unstable with the formation of what appears to be a helical vortex. The wavelength of the instability $\simeq 3 \bar{\delta}$ where $\bar{\delta}$ is the average boundary layer thickness, the average being taken over the time the flow is unstable. The time of formation of the vortices scales with the average convective time scale and is $\simeq 33 /(\overline{\Delta u / \delta})$, where $\Delta u=\left(u_{\max }-u_{\min }\right)$ and $u_{\max }, u_{\min }$ and $\delta$ are the maximum velocity, minimum velocity and boundary layer thickness respectively at each instant of time. The time to transition to turbulence is $\simeq 39 /(\overline{\Delta u / \delta})$. Quasi-steady linear stability analysis of the velocity profiles brings out two important results. First that the stability characteristics of velocity profiles with reverse flow near the wall collapse when scaled with the above variables. Second that the wavenumber corresponding to maximum growth does not change much during the instability even though the velocity profile does change substantially. Using the results from the experiments and the stability analysis, we are able to explain many aspects of transition in oscillating pipe flow. We postulate that unsteady boundary layer separation at high Reynolds numbers is probably related to instability of the reverse flow region.

\section{Introduction}

Two types of unsteady viscous flow have received considerable attention in the literature. The first one is oscillatory flow (with zero mean) and pulsatile flow (with non-zero mean) over flat plates and in pipes. The second one is unsteady flow past a body, for example an impulsively started cylinder or oscillating airfoil. Pulsatile flow is important in the design of pumps and other process equipment with transient flow patterns during startup, shutdown and change of operating conditions. Blood flow in arteries and respiratory flow in trachea are unsteady and it is important to understand the process of transition in such flows. Dynamic stall, related to unsteady boundary 
layer separation, is of importance in various aerodynamic applications including aircraft with high manoeuvrability, helicopter rotors, wind turbines, and jet engine compressor blades. A common feature in both these types of flows is the existence of inflection-point velocity profiles (often with reverse flow) that are unsteady. It is well known that velocity profiles with inflection points are highly unstable. Our purpose in this paper is to understand the stability and transition to turbulence of such flows.

Unsteady boundary layer separation is not yet fully understood and there is no specific criterion for its occurrence, in contrast to steady separation. Under steady conditions, the boundary layer separation point is defined as the point at which the wall shear stress becomes zero. It is observed in practice that just downstream of this point streamlines diverge away from the body. In unsteady flows there is no such universally accepted criterion. Vanishing of wall shear stress does not have any special significance in unsteady flows. A thin region of reverse flow often exists just downstream of the zero wall shear stress point and the boundary layer approximation is valid until the flow eventually breaks away with sudden thickening of this region (Sears \& Tellinois 1975). The breakaway is termed unsteady separation and is invariably accompanied by formation of vortices. The unsteady separation process seems to be quite complex and the details depend on geometry (airfoil, cylinder, etc.), type of motion (impulsive, oscillatory) and Reynolds number. For example the structure of unsteady separation for an impulsively started cylinder clearly shows a Reynolds number dependence (Bouard \& Coutanceau 1980): at Reynolds numbers less than about 5000 the wake vortices grow by accumulation of the upstream boundary layer vorticity, whereas at a Reynolds number of 9500 a number of vortices develop in the thin reverse flow region before full separation occurs. One view of unsteady separation is that it can be considered to occur when a singularity in the boundary layer solution is observed (Van Dommelen \& Shen 1982). We, however, believe the initial formation of the vortices at least in some types of unsteady separation, as in the $R e=9500$ case, is a result of instability of the reverse flow region. The recent study of Cassel, Smith \& Walker (1996) shows that in the vicinity of the separation point (in unsteady boundary layer separation at high Reynolds number) the velocity profiles become unstable at the very onset of viscous-inviscid interaction, prior to the appearance of the singularity. Reviews and references for the extensive work done in unsteady separation may be found in Sears \& Tellinois (1975), Cowley, Van Dommelen \& Lam (1990), Puhak, Degani \& Walker (1995) and Cassel et al. (1996).

The other class of unsteady flows is oscillating flow with or without a superimposed mean velocity. The oscillating flow may be caused by an oscillating piston in a pipe (Hino, Sawamoto \& Takasu 1976; Akhavan, Kamm \& Shapiro 1991a; Ohmi et al. 1982; Merkli \& Thomann 1975), by a sinusoidally varying free-stream velocity over a flat plate (Collins 1963; Jensen, Sumer \& Fredsøe 1989), or by a longitudinal oscillation of a flat plate in still or moving fluid (Li 1954; Sarpkaya 1993). In all cases the velocity profile is unsteady, often with reverse flow. In spite of the vast amount of work done there are many unanswered questions regarding the stability and transition of such flows. Depending on the Reynolds number and frequency of oscillation a number of features, not fully explained, like disturbed laminar flow and intermittently turbulent flow have been observed (see e.g. Akhavan et al. 1991a). It is known that transition is due to instability of the inflection point profiles, but the exact nature of the transition process is not understood.

A related type of unsteady flow which has been studied is when the free stream over a flat surface has a constant acceleration or deceleration. Fales (1955) ob- 
served experimentally the formation of two-dimensional vortices and subsequent three-dimensionality when a flat plate moving in its plane in still fluid is abruptly stopped. Gad-el-Hak et al. (1984) in a similar experiment linearly decelerated a plate from some constant speed to a lower constant speed. The Reynolds numbers were low enough that during the constant speed phases of plate motion the flow was laminar. However, during the deceleration phase formation of inflection point profiles led to instability and transition to turbulence. Yang, Spalart \& Ferziger (1992) were able to reproduce these results using direct numerical simulation (DNS). Lefebvre \& White (1989) found abrupt transition to turbulence in an accelerating flow in a long pipe. The critical Reynolds number based on boundary layer thickness was about 25000 .

There is no satisfactory linear stability analysis for velocity profiles whose mean value is changing with time. Shen (1961) has carried out stability analysis for velocity profiles which are unsteady, but similar. Two common methods for stability analysis of unsteady flows have been the quasi-steady approximation and Floquet theory, which is only appropriate for periodic flows. Seminara \& Hall (1975) and Hall \& Parker (1976) have shown that the quasi-steady approximation is fairly accurate as long as disturbances grow at a rate fast compared to the rate of change of the mean flow. The quasi-steady approximation has been satisfactorily used to explain observed instability in many unsteady situations (Thorpe 1971; Obremski \& Fejer 1967). Direct numerical simulation (Akhavan, Kamm \& Shapiro 1991b) of oscillating pipe flow shows that infinitesimal disturbances evolve as would be predicted by quasi-steady stability analysis. Nonlinearity and secondary instability come into the picture only at later times and are essential to describe the final stages of transition. A straightforward application of stability theory, however, still does not answer many questions. For example in oscillating pipe flow stability theory predicts a critical Reynolds number (based on Stokes layer thickness) of 82 whereas the experimentally observed value is in the range of 300 to 500; and turbulent bursts are observed during the deceleration phase whereas stability theory predicts the most unstable velocity profile to occur during the start of the acceleration phase. Oscillating pipe flow is discussed in more detail in $\S 5.5$.

\section{Present work}

In the situations discussed above, analysis or explanation of the physical process is difficult. Dynamic stall depends on too many parameters: the pressure gradient changes both in space and time. In the case of oscillatory pipe flow the effect of the acceleration or deceleration phases cannot be studied separately. The turbulence or perturbations generated during one phase can change the flow in the rest of the cycle.

Therefore in the present work we have developed a novel experimental technique to study unsteady velocity profiles with reverse flow. The principle of these experiments is illustrated below. We generate motion in a long pipe with a piston. The piston accelerates from zero velocity to $U_{p}$ for time $0<t<t_{0}$, maintains constant velocity for $t_{0}<t<t_{1}$, decelerates to zero velocity for $t_{1}<t<t_{2}$, and is stationary for $t>t_{2}$. A typical variation of piston velocity with time is shown in figure 1(a). The velocity profiles during the phases are shown in figure $1(b)$. A velocity profile with reverse flow close to the wall develops for $t>t_{1}$. These velocity profiles are unsteady and have inflection points, and hence become unstable at relatively low Reynolds numbers. The instability and evolution of such flows is the main focus of this study.

Weinbaum \& Parker (1975) have done related work. They have investigated theoretically the laminar decay of a fully developed channel or pipe flow following a 


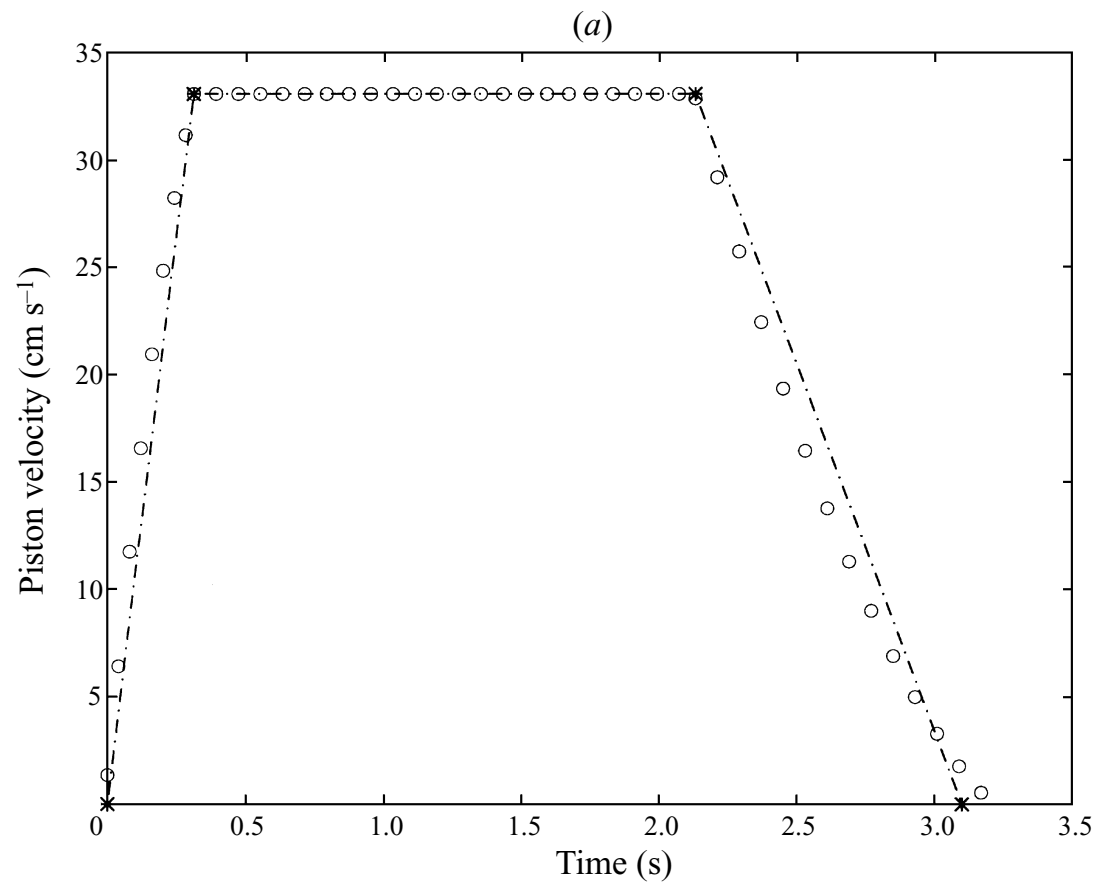

(b)
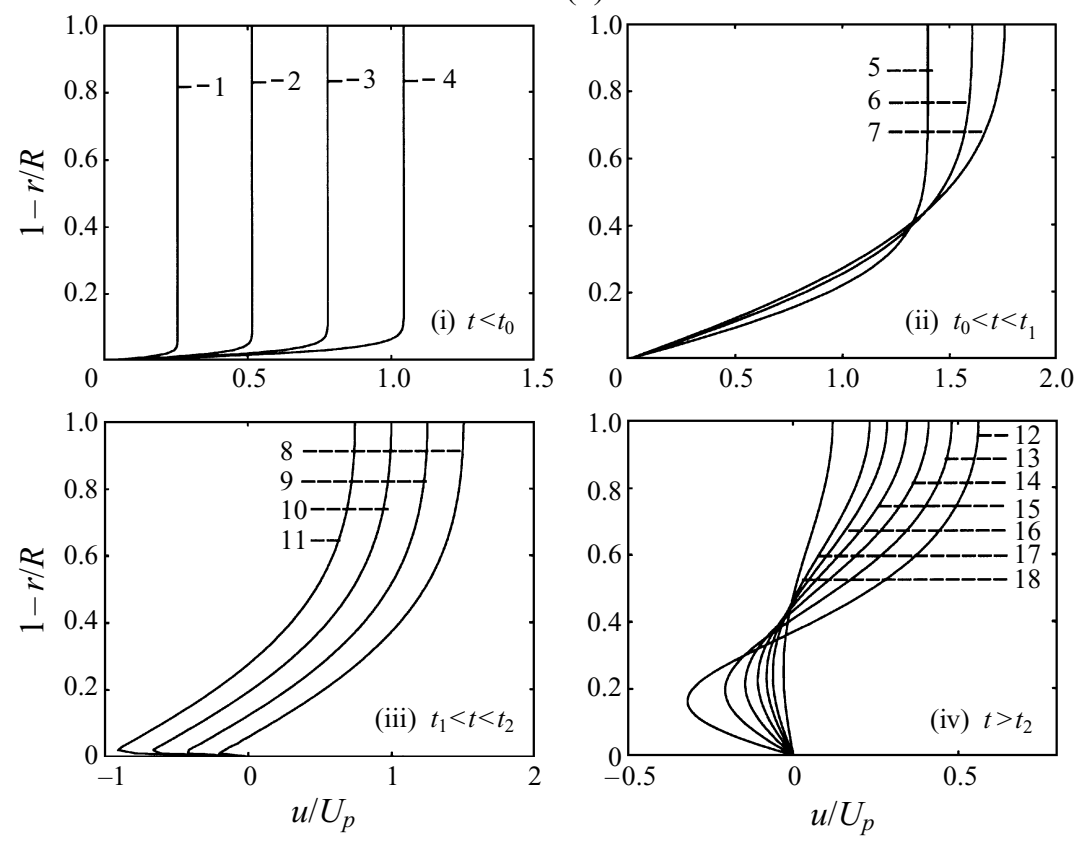

Figure 1. (a) Typical variation of piston velocity with time; $t_{0}$ is the time of acceleration, $t_{1}$ is the time when stepper motor is switched off and $t_{2}$ is the time when piston motion stops. Circles represent the actual piston velocity and the dashed-dotted line is the assumed trapezoidal motion. (b) Velocity profiles for different times, (i) during the acceleration of the piston; curve $1, t=t_{0} / 4 ; 2$, $t=2 t_{0} / 4 ; 3, t=3 t_{0} / 4 ; 4, t=t_{0}$; (ii) when piston velocity is constant; curve $5, t=t_{0}+\left(t_{1}-t_{0}\right) / 3 ; 6$, $t=t_{0}+2\left(t_{1}-t_{0}\right) / 3 ; 7, t=t_{1}$ (iii) during the deceleration of the piston; curve $8, t=t_{1}+\left(t_{2}-t_{1}\right) / 4$; $9, t=t_{1}+2\left(t_{2}-t_{1}\right) / 4 ; 10, t=t_{1}+3\left(t_{2}-t_{1}\right) / 4 ; 11, t=t_{2} ;$ and (iv) after the piston motion stops; curve $12, t=t_{2}+\left(t_{p}-t_{2}\right) / 6 ; 13, t=t_{2}+2\left(t_{p}-t_{2}\right) / 6 ; 14, t=t_{2}+3\left(t_{p}-t_{2}\right) / 6 ; 15, t=t_{2}+4\left(t_{p}-t_{2}\right) / 6$ $16, t=t_{2}+5\left(t_{p}-t_{2}\right) / 6 ; 17, t=t_{p}$; and $18, t=t_{v}=23.6$ s. $U_{p}$ refers to constant piston velocity. 
Stepper motor

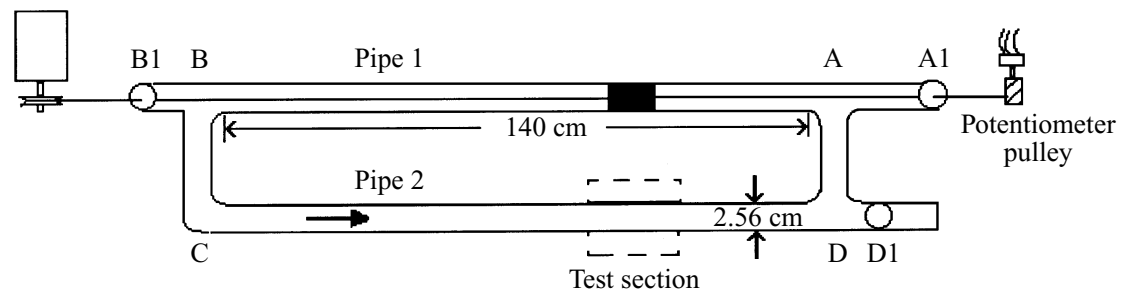

FIGURE 2. Schematic representation of experimental setup.

sudden blockage. The pressure wave passes at the speed of sound, resulting in reverse flow near the wall immediately after the blockage of the channel or pipe, to satisfy the zero mass flow condition. Weinbaum \& Parker (1975) obtain the velocity profiles approximately by solving the one-dimensional unsteady governing equation using the Pohlhausen type technique. We, however, obtain exact solutions for the velocity profiles with time for any given piston motion.

The paper is organized as follows. The experimental work is described in $\S 3$. The analytical solution is discussed in section 4 with details given in the Appendix. In $\S 5$ we present the main results of this study. Transition of oscillatory pipe flow and unsteady separation of the boundary layer are discussed in relation to these results in the same section. Section 6 concludes the paper.

\section{Experiments}

\subsection{Experimental setup}

The experimental setup is shown in figure 2. The arrangement is a closed loop, clamped to a wooden board. Glass pipes of $2.56 \mathrm{~cm}$ diameter and $145 \mathrm{~cm}$ long form two legs of the loop, connected with PVC pipes at either end. Pipe 1 contains a piston while pipe 2 contains the test section. One end of the piston is connected through an inextensible string to a stepper motor; the other end of the piston is simultaneously connected to a ten turn potentiometer. The output signal from the potentiometer is stored in an oscilloscope and is subsequently used to get piston velocity as a function of time. The light from a light emitting diode, connected with the stepper motor controller and kept below the test section, is recorded by a video camera simultaneously with the flow structure. This signal gives time of starting of the motor and the time when the motor is switched off. In all cases, the length of the piston motion was such that disturbances from the bends did not convect to the test section during the time of the experiment. The deceleration times are of the order of $0.01 \mathrm{~s}$ to $0.5 \mathrm{~s}$, usually much smaller than the transition time which is of the order of $1 \mathrm{~s}$ to $10 \mathrm{~s}$. The acceleration and deceleration phases are well approximated by linear changes of velocity with time.

We have also done a few experiments in a channel with rectangular cross-section $(15 \times 5.4 \mathrm{~cm})$ and $212 \mathrm{~cm}$ long. The rest of the arrangement is similar to the pipe-flow setup.

\subsection{Flow visualization}

In all the experiments water was the working fluid. We injected fluorescent dye at the top or bottom wall along the pipe in bands of approximately $5 \mathrm{~mm}$ width before the start of each experiment; in some of the experiments we had dye on both the 
top and bottom walls of the pipe. Similiarly dye was injected on the top and bottom walls in the channel experiments. The top dye density was $0.5 \mathrm{~kg} \mathrm{~m}^{-3}$ less than the density of the working fluid; similiarly the bottom dye density was $0.5 \mathrm{~kg} \mathrm{~m}^{-3}$ more than the working fluid density. The density differences were created by adding appropriate amounts of common salt. This density difference ensured that the two dye lines remain at the top and bottom of the pipe/channel before the start of the motor. The effect of this density difference is negligible as the Richardson number $\left(R_{i}=g(\Delta \rho / \rho)\left(R / U_{p}^{2}\right)\right)$ is less than 0.02 for all the experiments. Dye was injected by inserting two flexible tubes containing the dyes. These tubes were inserted to the end $\mathrm{C}$ of pipe 2 and dye was injected slowly and the flexible tubes pulled out of pipe 2 simultaneously. The motion produced due to the dye injection was allowed to settle down to rest for about five minutes before the experiment was started. A $3 \mathrm{~mm}$ thick vertical sheet of light aligned with the flow from a $1000 \mathrm{~W}$ halogen lamp was passed through the test section. Motion of the dye was recorded on a video camera placed perpendicular to the plane of the light sheet. The starting and switching off of the motor was recorded by the light emitting diode connected to the motor switch and kept in the field of view of the camera.

In some of the experiments we used polystyrene particles (specific gravity $=1.05$ ) of size less than 100 microns to visualize the flow. Fluid and particle densities were matched by adding salt to the water. Flow was recorded on video tape and long exposure photographs with a still camera were taken from the video recording to get the structure of the flow. From particle visualization it was confirmed that the flow is unidirectional during the acceleration phase and during the deceleration phase until the instability sets in.

From dye visualization pictures we observe how the flow becomes unstable. The dye line takes the form of a wave (see figures 3-8) some time after the piston motion stops and at a later time a series of vortices appears. We measure the time when a perceptible wave appears $\left(t_{p}\right)$. This indicates the time when the instability sets in and the flow ceases to be unidirectional. The waves can be detected only when the amplitude of the waves is approximately $1 \mathrm{~mm}$. The dye line subsequently rolls up into spirals. The beginning of the rollup process is taken as the time of appearance of the vortex $\left(t_{v}\right)$ and the distance between adjacent vortices or wavelength of the instability, $\lambda$, is also measured. Estimated errors in the measurement of $t_{p}$ and $t_{v}$ and $\lambda$ is $5 \%$.

\subsection{Hot-wire measurements}

Hot-wire measurements obtained using a constant temperature anemometer (DANTEC 56C01 CTA with 56C17 CTA bridge) were mainly used to get the transition to turbulence times. The probe mounted on a long stem and inserted from one end of the pipe was nearly parallel to the pipe axis. The wire was placed such that it never encountered reverse flow so as to ensure there was no contamination of the signal due to the probe stem. We also used a wedge-shaped hot-film probe (DANTEC 55R32) which however did not have sufficient frequency response to indicate transition.

\subsection{Range of parameters}

Two parameters that can be varied in our experiment are the piston speed and the piston travel distance. We varied maximum piston speeds from $5.35 \mathrm{~cm} \mathrm{~s}^{-1}$ to $47.3 \mathrm{~cm}$ $\mathrm{s}^{-1}$ and the piston travel distance was between $8 R$ and $56 R$ in the pipe. The thickness of the boundary layer $\left(\delta_{1}\right)$ when the motor is switched off depends on the distance travelled by the piston for a particular speed. The Reynolds number $\left(R e_{\delta_{1}}\right)$ based 
on the boundary layer thickness $\delta_{1}$ and the centreline velocity at $t_{1}\left(u_{\max 1}\right)$ ranges from 250 to 3000 and the range of $\delta_{1} / R$ is between 0.1 and 0.91 . The boundary layer thickness was calculated using the analytical solution described in the next section. The assumption in the calculation is that the flow is unidirectional and laminar which was confirmed by particle flow visualization; instability, for the Reynolds numbers achieved in our experiments, always occurs after the piston deceleration has started.

\section{Analytical solution}

We consider axisymmetric, unsteady, incompressible flow in an infinitely long pipe. Let $r$ be the radial distance from the centre of the pipe, $\theta$ the azimuthal angle and $x$, the axial distance. From the experiments it is observed that the flow remains unidirectional (unidirectional is taken to mean existence of velocity in the $x$-direction only and includes reverse flow) for a considerable time and hence we look for the solution which is parallel to the $x$-direction. The absence of radial and azimuthal velocities implies that the velocity in the $x$-direction $u$ is function of radial coordinate $r$ and time $t$ only. With these approximations the governing equation of motion in the $x$-direction becomes

$$
\frac{\partial u}{\partial t}=-\frac{1}{\rho} \frac{\partial p}{\partial x}+v\left(\frac{\partial^{2} u}{\partial r^{2}}+\frac{1}{r} \frac{\partial u}{\partial r}\right)
$$

and the radial momentum equation becomes

$$
\frac{\partial p}{\partial r}=0 .
$$

Thus the axial pressure gradient, $\partial p / \partial x$ is a function just of time.

Boundary conditions are

$$
u(R, t)=0, \quad \frac{\partial u(0, t)}{\partial r}=0
$$

and initial condition is

$$
u(r, 0)=0 .
$$

Solution of these equations is possible if pressure as a function of time is known. In our case, however, pressure is unknown and determined indirectly by the piston motion. Assuming incompressible flow, the piston motion is felt immediately at each cross-section of the pipe and the volume flux at any cross-section corresponds to the volume flux due to piston motion,

$$
\int_{0}^{R} 2 \pi r u \mathrm{~d} r=u_{p}(t) \pi R^{2}
$$

where $u_{p}$ is the piston velocity.

With this additional condition, analytical solution of equation (4.1) is possible. We solve it using the Laplace transform technique. The details of the solution for the axisymmetric case are given in Appendix A. Solution for the channel flow is given in Appendix B. The solution depends on $t_{0}, t_{1}$ and $t_{2}$. Velocity profiles calculated using the procedure are shown in figure $1(b)$ for different times for a maximum piston speed $\left(U_{p}\right)$ of $5.35 \mathrm{~cm} \mathrm{~s}^{-1}$ and $t_{0}, t_{1}, t_{2}$ and $t_{p}=0.13,10.26,10.27$ and $19.2 \mathrm{~s}$ respectively. The centreline velocity changes with the piston velocity during the acceleration phase and the boundary layer near the wall starts growing. When the piston moves at constant 


\begin{tabular}{lrrrrrrrrrr} 
Case & $t_{0}(\mathrm{~s})$ & $t_{1}(\mathrm{~s})$ & $t_{2}(\mathrm{~s})$ & $t_{p}(\mathrm{~s})$ & $t_{v}(\mathrm{~s})$ & $U_{p} \mathrm{~cm} \mathrm{~s}^{-1}$ & $R e=\frac{U_{p} R}{v}$ & $\frac{\delta_{1}}{R}$ & $R e_{\delta_{1}}$ \\
I & 0.13 & 10.26 & 10.27 & 19.2 & 23.6 & 5.35 & 684.0 & 0.85 & 1023.5 \\
II & 0.42 & 3.68 & 4.04 & 6.24 & 6.92 & 15.91 & 2036.5 & 0.55 & 1558.6 \\
III & 0.14 & 1.86 & 2.46 & 2.76 & 3.20 & 33.21 & 4251.0 & 0.39 & 2104.3 \\
IV & 0.13 & 0.44 & 1.10 & 1.80 & 2.56 & 33.21 & 4251.0 & 0.18 & 852.9 \\
\multicolumn{8}{c}{ TABLE 1. Parameters of the experiments. }
\end{tabular}

velocity, due to diffusion of vorticity, the boundary layer grows with time and the centreline velocity increases to meet the mass flow condition. When the motor is switched off the piston decelerates and stops at time $t_{2}$. During piston deceleration the velocity profile shifts to satisfy the flux condition with a thin layer growing near the wall to satisfy the no-slip condition. During this phase the pressure gradient is large and adverse. After the piston motion ceases the velocity profiles continue to show reverse flow near the wall to satisfy zero mass flow condition; with time the velocity decays to zero everywhere. There is generally a slight adverse pressure gradient but most of the changes in the velocity profiles are due to diffusion. Velocity profiles beyond $t_{1}$ contain an inflection point and have reverse flow. The solution is clearly valid only as long as the fluid velocity is parallel to the pipe axis, i.e. till before the flow becomes unstable and three-dimensional.

To our knowledge an analytical solution for arbitrary piston motion has not been reported in the literature. Weinbaum \& Parker (1975) have solved similar equations to obtain the solution for unidirectional flow when a fully developed flow in a channel or a pipe is blocked impulsively. Their solution, however, is approximate and is obtained using a Pohlhausen type technique used in boundary layer calculations.

\section{Results and discussion}

\subsection{Flow visualization results}

A common feature we observe in our experiments is that the flow becomes unstable sometime after the motor is switched off. The instability is accompanied by the formation of a periodic array of vortices. The time of formation of the vortices and the spacing between them depends on the experimental conditions. We choose four representative cases from our experiments in the pipe to describe the main features. The parameters are given in table 1 . The flow visualization results for these cases are shown in figures 3 to 8 . In the cases where flow is visualized on only one of the walls, the centreline is marked by a white line. The time when the motor was switched on is taken as zero time.

Figure 3 shows the flow evolving with time for case I $\left(R e_{\delta_{1}}=1023.5\right.$ and $\delta_{1} / R=0.85$ ). (Recall subscript 1 refers to the time when the motor is switched off.) The dye line takes the form of a wave at $t \approx 19.2 \mathrm{~s}$. (The non-dimensional time $t^{*}=\left(t-t_{1}\right) /\left(t_{p}-t_{1}\right)$, is also given for each photograph in the caption.) Flow visualization with particles shows that the flow is wavy even away from the wall. At $t \approx 23.6 \mathrm{~s}$ discrete vortices are formed. These vortices grow with time and move in the direction of the initial flow. The picture at $t \approx 42 \mathrm{~s}$ (figure $3 d$ ) corresponds to a situation when all motion has stopped; the dye pattern seen is an integrated effect of previous motions. At these low Reynolds numbers the flow never becomes turbulent. 


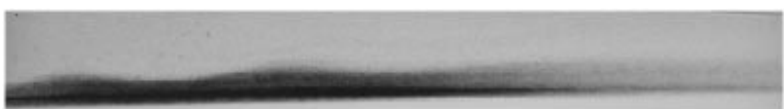

(a)

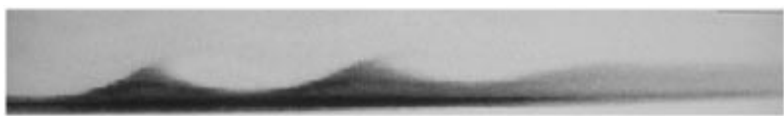

(b)

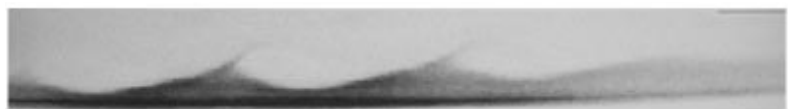

(c)

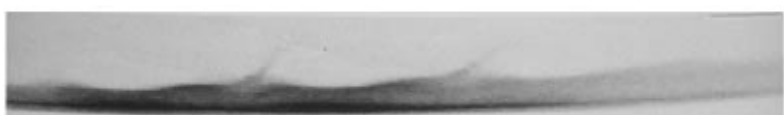

(d)

FIGURE 3. Flow visualization pictures of case I showing the development of the dye line initially present at the bottom of the pipe. Initial direction of fluid motion is from left to right. (a) $t=21.64$ $\mathrm{s}$ which corresponds to $t^{*}=\left(t-t_{1}\right) /\left(t_{p}-t_{1}\right)=1.27,(b) t=27.64 \mathrm{~s}, t^{*}=1.94,(c) t=36.64 \mathrm{~s}$, $t^{*}=2.95$ and $(d) t=41.64 \mathrm{~s}, t^{*}=3.51$.

Case II corresponds to $R e_{\delta_{1}}=1558.6$ and $\delta_{1} / R=0.55$ with the instability waves formed at $t \approx 6.24 \mathrm{~s}$ and the vortices at $t \approx 6.92 \mathrm{~s}$. Figure 4 shows the dye structure at several times after the vortices have formed. Flow is visualized on both the top and bottom of the pipe. It is important to note the difference in phase of the periodic vortices at the top and bottom. The phase difference is approximately $180^{\circ}$. This is only possible if the vortices at the top and bottom of the pipe are part of a single helical vortex. We observe this $180^{\circ}$ phase difference between the bottom and top vortices in experiments where the initial boundary layer thickness $\left(\delta_{1}\right)$ is not small compared to the radius of the pipe. It is a key finding of the present study. This suggests that a helical mode of disturbance is the most unstable. In the channel the antisymmetric mode of disturbance was found to be the most unstable one.

At later times there appears to be a strong interaction between the vortices, which distorts them (figure $4 c$ ). Dye from both walls moves across the pipe centreline towards the opposite wall (figure 4d). At $12.64 \mathrm{~s}$, the vortices break down and then rapid mixing of the dye occurs suggesting transition to turbulence. Note that the centres of the vortices do not move in the axial direction, unlike in case I.

Figure 5 (case III) shows the flow structure for $R e_{\delta_{1}}=2104.3$ and $\delta_{1} / R=0.39$. Only the top half of the pipe is shown. The spacing between the vortices has decreased compared to the previous two cases. Unlike in case II the vortices are closer to the wall and there seems to be less interaction - there is no distortion of the vortices. We also observe a feature not present in the previous cases, a secondary vortex close to the wall induced by the primary vortex. The secondary vortex cannot be seen in the pictures shown in figure 5 because of the present technique of flow visualization. The secondary vortices are more clear in particle visualization pictures. The primary vortices move away from the wall as the secondary vortices grow. The breakdown to turbulence is very rapid (it occurs between $t \approx 3.88$ and $4.00 \mathrm{~s}$ ). While viewing the video we can readily appreciate the rapidity of the breakdown process. The transition process could be similar to the mixing transition in shear layers. Mixing transition occurs with 


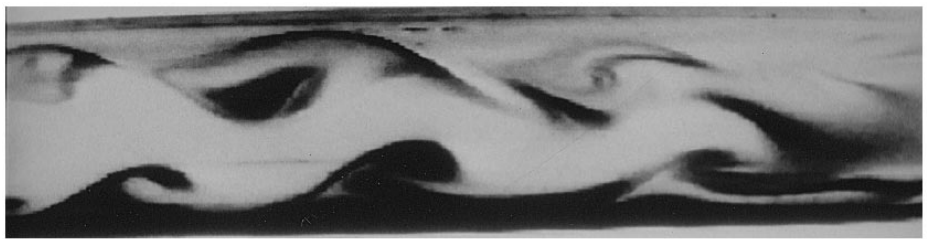

(a)

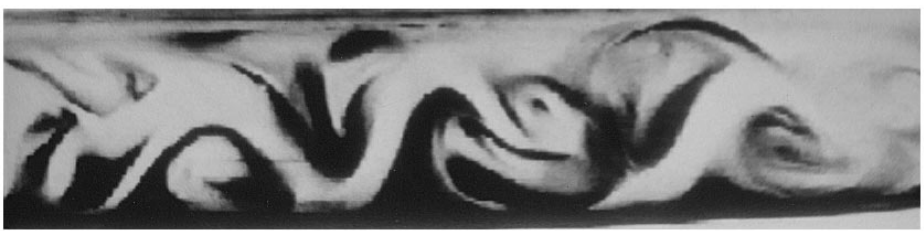

(b)

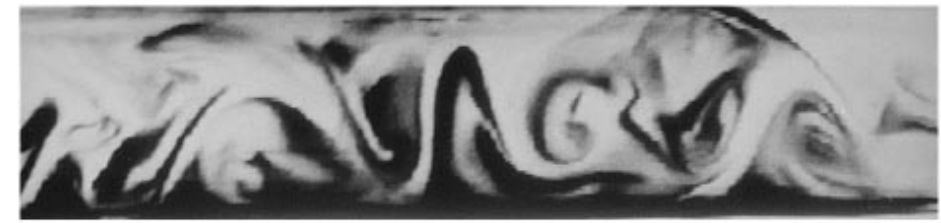

(c)

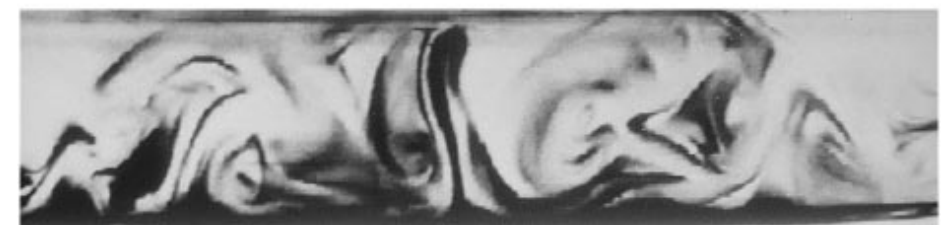

(d)

FIGURE 4. Flow visualization pictures of case II showing the development of the dye line initially present at both the top and bottom of the pipe. Initial direction of fluid motion is same as in figure 3. (a) $t=9.88 \mathrm{~s}, t^{*}=2.42$, (b) $t=11.80 \mathrm{~s}, t^{*}=3.17$, (c) $t=12.64 \mathrm{~s}, t^{*}=3.5$ and $(d) t=13.64 \mathrm{~s}$, $t^{*}=3.89$.

the appearance of three-dimensionality in the primary Kelvin-Helmoltz vortices and is accompanied by rapid mixing of the two streams. Recently Schoppa, Hussain \& Metcalfe (1995) have sought to explain the phenomenon on the basis of vortex core dynamics. Akhavan et al. (1991b) also show, by numerical simulation, that the transition to turbulence in oscillating pipe flow can be explained by secondary instability of twodimensional finite-amplitude waves. Sarpkaya (1993) has detected a variety of coherent structures like low-speed streaks and hairpin eddies near the wall during transition in oscillating Stokes flow. Presumably similar structures occur in our experiments as well.

We generally observe the secondary vortices at the higher Reynolds numbers. An example of the formation and growth of the secondary vortices is shown in figure 6 for $R e_{\delta_{1}}=1521.4$ and $\delta_{1} / \mathrm{R}=0.39$. Gradual growth of this secondary vortex and its movement towards the centre is clear from the subsequent pictures. Secondary vortices are common in many situations, for example during impact of a vortex ring against a wall (Walker 1978), and the interaction of a line vortex with a wall (Pullin \& Perry 1980).

In figure 7 (case IV) the piston speed is same as the case III, but the piston travels a lesser distance. $R e_{\delta_{1}}$ and $\delta_{1} / R$ are 852.9 and 0.18 respectively. In this case 


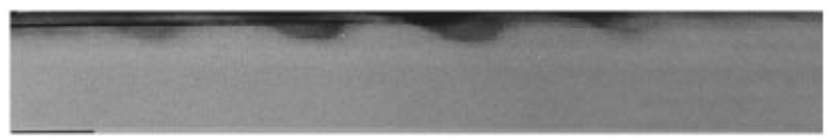

(a)

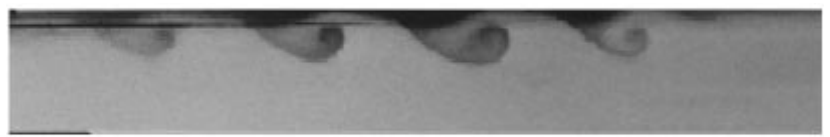

(b)

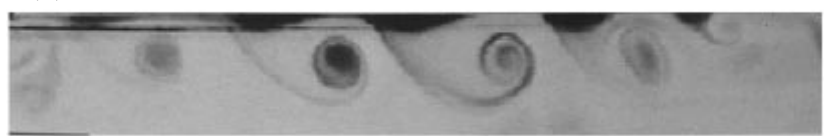

(c)

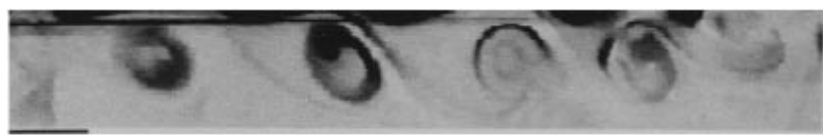

$(d)$

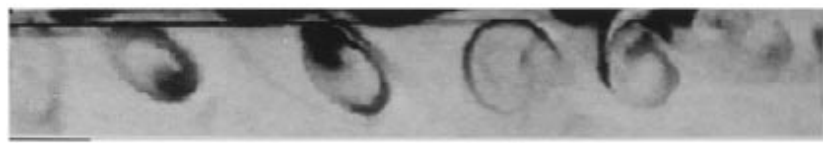

(e)

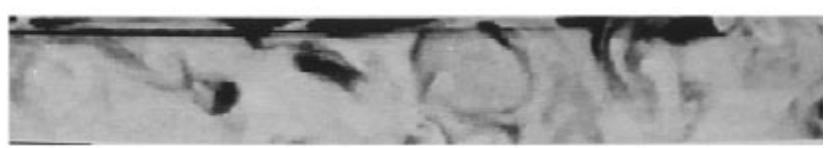

$(f)$

Figure 5. Same as figure 3 for case III except that the initial dye line is present at the top of the pipe. (a) $t=3.20 \mathrm{~s}, t^{*}=1.49$, (b) $t=3.40 \mathrm{~s}, t^{*}=1.71$, (c) $t=3.72 \mathrm{~s}, t^{*}=2.07$, (d) $t=3.88 \mathrm{~s}$, $t^{*}=2.24,(e) t=3.96 \mathrm{~s}, t^{*}=2.33$ and $(f) t=4.28 \mathrm{~s}, t^{*}=2.69$.

vortices do not break down to make the flow turbulent and the vortical disturbances remain confined near the wall. Movement of the vortices in the horizontal direction is observed. Note that the $R e_{\delta_{1}}$ values of case I and case IV are comparable and in both these cases we do not observe breakdown to turbulence.

In many of the experiments, especially when $\delta_{1} / R<0.4$, the waves/vortices do not form simultaneously on the top and bottom walls (figure 8 ). We do not have an explanation for this observation. In these cases it is also not possible to ascertain whether the phase difference between top and bottom wall vortices is $180^{\circ}$, i.e. whether the mode is helical.

We observe similar features in preliminary experiments in a channel with rectangular cross-section. Phase differences of $180^{\circ}$ between top and bottom vortices are observed when $\delta_{1} / H$ is greater than about 0.4 , i.e. the antisymmetric mode is most unstable. Here $H$ is the half-height of the channel. For $\delta_{1} / H$ lesser than about 0.4 the growth of the vortices at the top and bottom seems to be independent of each other.

\subsection{Hot-wire measurements}

The hot-wire and the hot-film measurements are mainly used to get the time, $t_{p h}$, at which flow ceases to be unidirectional and the time $t_{t r}$ when the transition to turbulence occurs. Figure 9 shows two traces on an oscilloscope from a hot-wire signal. One trace shows the total voltage (d.c. coupled) and the other the fluctuating 
(a)
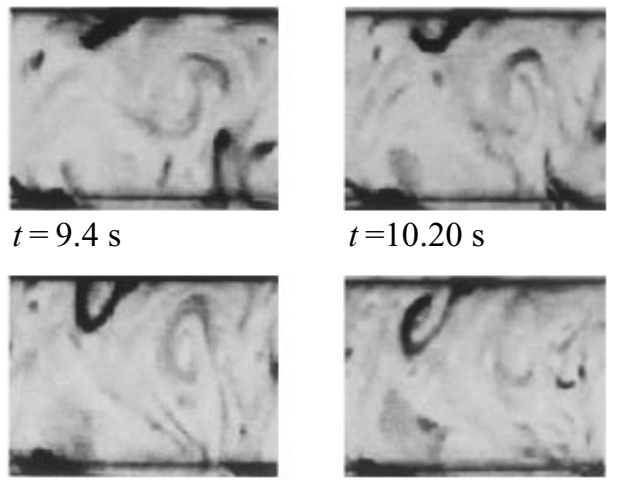

$t=10.20 \mathrm{~s}$

$t=11.44 \mathrm{~s}$

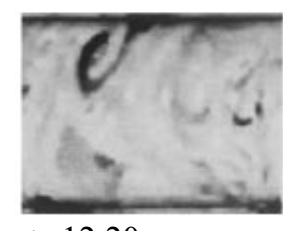

$t=12.20 \mathrm{~s}$

(b)
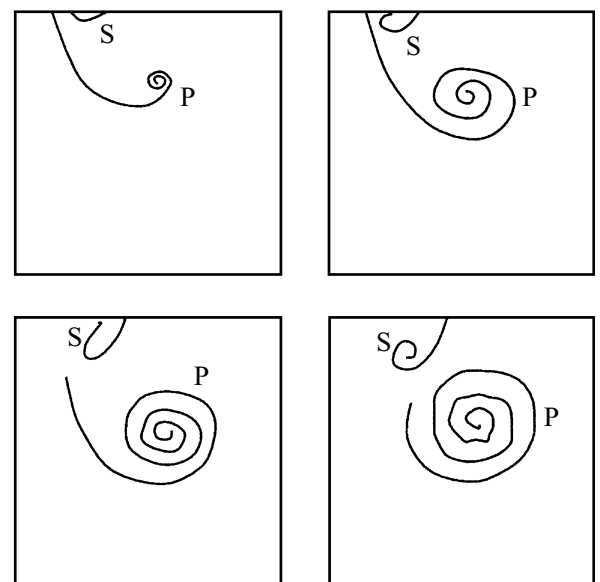

FIGURE 6. (a) Flow visualization and $(b)$ schematic, showing formation of a secondary vortex at $R e_{\delta_{1}}=1521.4$ and $\delta_{1} / R=0.39$ for $t=9.4 \mathrm{~s}, 10.2 \mathrm{~s}, 11.44 \mathrm{~s}$ and $12.2 \mathrm{~s}\left(t^{*}=4.32,4.77,5.48\right.$ and 5.91 respectively), The primary vortex (marked $\mathrm{P}$ ) is anticlockwise and the secondary vortex (S) is clockwise. $t_{0}, t_{1}$ and $t_{2}$ for this experiment are $0.12 \mathrm{~s}, 1.8 \mathrm{~s}$ and $2.42 \mathrm{~s}$ respectively.

part only (a.c. coupled). In the d.c. coupled trace we can identify three regimes after the motor is switched off: a sharp reduction in the voltage due to the adverse pressure gradient, a slower reduction in velocity due to diffusion and finally again a larger reduction in voltage due to the formation of the instability vortices. We take the time at which the second change in curvature occurs as $t_{p h}$. We observed that, as expected, within the experimental scatter $t_{p h} \simeq t_{p}$. Transition is clearly indicated in the a.c. coupled signal by a high-frequency fluctuation, characteristic of turbulence. Repeated experiments with the same piston-velocity time histories show some scatter in the observed transition times. The change in position of the vortices relative to the hot wire with each experiment seems to cause this scatter. (This type of difference is also seen in the hot-wire traces of Hino et al. (1976) in oscillating pipe flow: the phase at which breakdown occurs changes from cycle to cycle for the same experimental conditions.) In the results presented in figure $15(c)$ we have taken $t_{t r}$ to be the minimum value from at least five experiments. Note that the turbulence is dissipated quite rapidly. At the lower Reynolds numbers only a single spike is observed. 


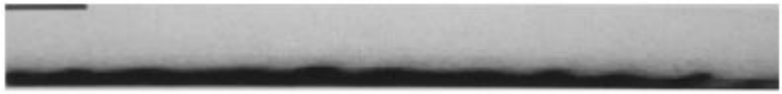

(a)

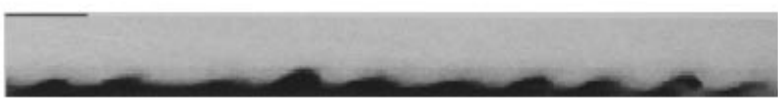

(b)

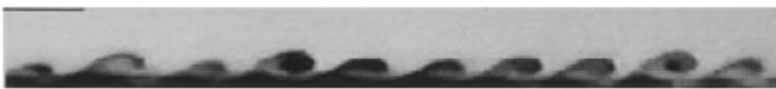

(c)

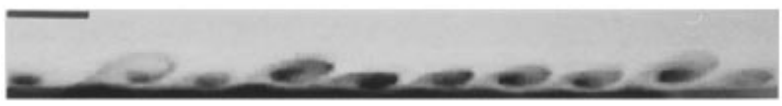

(d)

FIGURE 7. Same as figure 3 for case IV. (a) $t=2.08 \mathrm{~s}, t^{*}=1.21$, (b) $t=2.72 \mathrm{~s}, t^{*}=1.68$, (c) $t=3.84 \mathrm{~s}, t^{*}=2.5$ and $(d) t=5.44 \mathrm{~s}, t^{*}=3.68$.

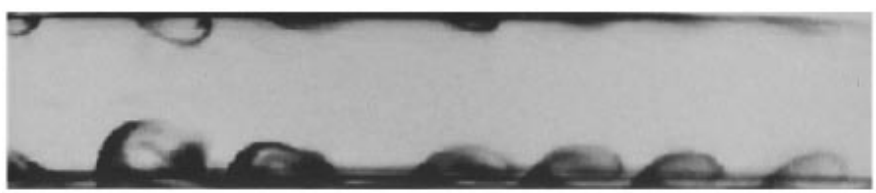

(a)

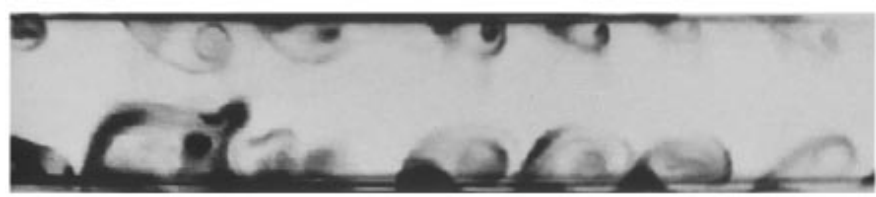

(b)

FIGURE 8. Flow visualization pictures for $R e_{\delta_{1}}=2146.1, \delta_{1} / R=0.35, t_{0}=0.16 \mathrm{~s}, t_{1}=1.52 \mathrm{~s}$ and $t_{2}=2.53 \mathrm{~s}$ Note that waves/vortices do not appear simultaneously on the top and bottom of the pipe walls at $t=2.92 \mathrm{~s}\left(t^{*}=1.17\right)(a)$. It can be observed that at $t=3.12 \mathrm{~s}\left(t^{*}=1.33\right)(b)$ the vortices only at the bottom wall have broken down. Initial direction of fluid motion is same as in figure 3 .

We remark that $t_{t r}>t_{v}$ and vortex formation does not always lead to turbulence; transition occurs only if the Reynolds number is high enough. Cases I and IV are examples where dye visualization shows vortex formation but does not show transition to turbulence. We found no transition to turbulence for $R e_{\delta_{1}} \leqslant 1200$.

In summary we note the following main observations :-

(i) Instability of the inflectional point profiles, as in the case of Kelvin-Helmoltz instability, results in the formation of a series of vortices. In the cases we have studied, the critical $R e_{\delta_{1}}$ below which the flow does not become unstable is about 500 .

(ii) Nearly periodic arrays of vortices appear in both the top and bottom half of the pipe (and channel) as the instability develops. When the boundary layer thickness is $>0.4$ there is a phase difference between the position of the top and bottom vortices. Therefore, in the pipe, what appears as discrete vortices in the flow visualization pictures is possibly a single helical vortex. 


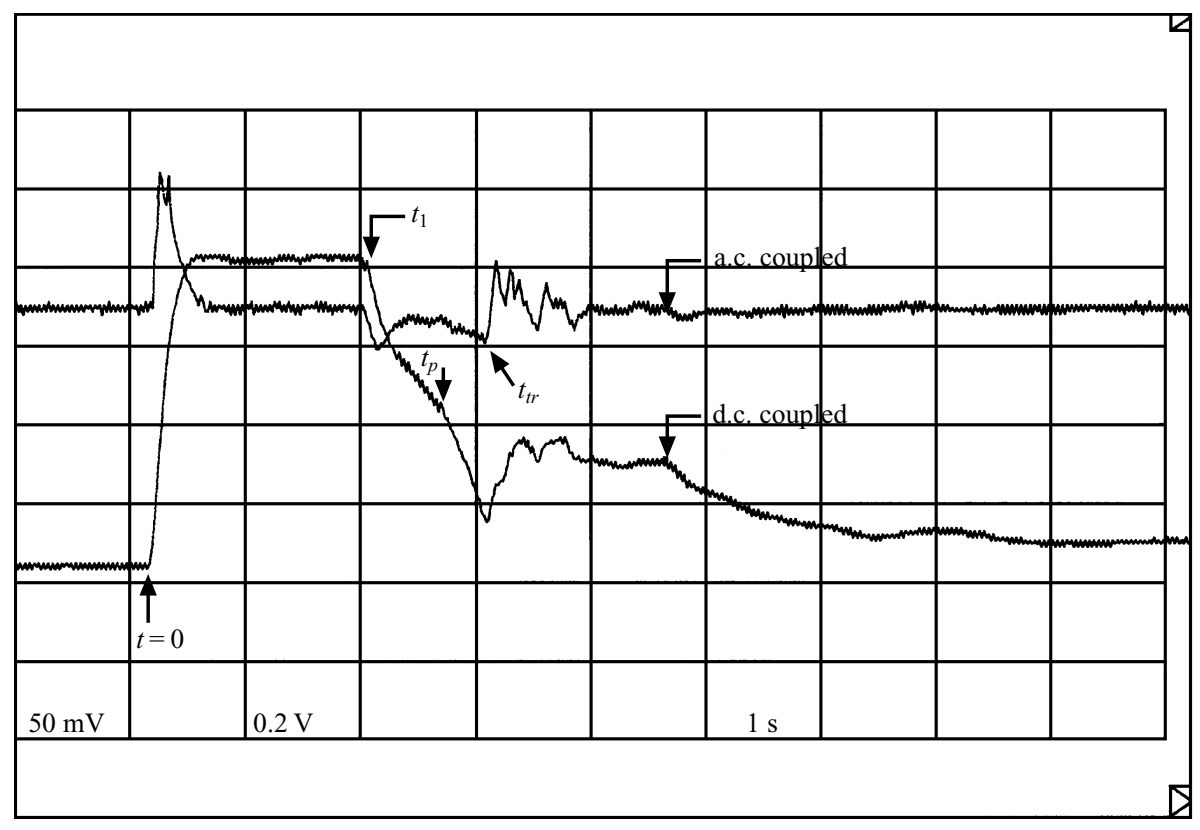

FIGURE 9. Traces of velocity variation for $R e_{\delta_{1}}=2065, \delta_{1} / R=0.34, t_{0}=0.4 \mathrm{~s} t_{1}=1.86 \mathrm{~s}$ and $t_{2}=1.98 \mathrm{~s}$, illustrating the time of development of the two/three-dimensional flow $\left(\right.$ at $\left.t_{p h}\right)$ and the transitional flow regime (at $t_{t r}$ ).

(iii) For $R e_{\delta_{1}}$ smaller than a critical value $(\simeq 1200)$ as in cases I and IV, the vortices do not break down to turbulence. When they do break down (case II and case III) the transition is very rapid.

(iv) When vortices are formed away from the wall they grow with time and move towards the centre of the pipe. There is strong interaction between the vortices which results in distortion and the final breakdown to turbulence.

(v) Secondary vortices are observed at the higher $R e_{\delta_{1}}$.

\subsection{Analyses}

To get an understanding of the instability process we next look at how some quantities of interest, like the velocity profiles, Reynolds number, and linear stability characteristics, vary with time. Figure 10 shows the calculated velocity profiles from the time the piston starts decelerating till the time the vortices are first seen for the four cases. (Any calculation beyond this time will be meaningless as the flow will no longer be unidirectional.) As discussed in $\S 4$, the changes in velocity during the piston deceleration $\left(t_{1}<t<t_{2}\right)$ are mostly due to the pressure gradient and the changes after the piston has stopped $\left(t>t_{2}\right)$ are mostly due to diffusion. The rapid changes in maximum velocity $\left(u_{\max }\right)$ and minimum velocity $\left(u_{\min }\right)$ due to the pressure gradient and the slower changes due to diffusion are clearly seen in figure 11, which shows the variation of $u_{\max } / U_{p}$ and $u_{\min } / U_{p}, R e_{\delta}$ (local Reynolds number $=\left(u_{\max }-u_{\min }\right) \delta / v=\Delta u \delta / v$ ), $\delta_{i}$ (location of the inflection point) and $R e_{\delta c r}$ (critical Reynolds number). It is clear in all cases that the velocity profiles change substantially during the development of the instability. It may be noted that even though the Reynolds numbers are high, diffusion is not negligible during the time the instability develops. Figure 11 also shows that $\operatorname{Re}_{\delta}$ continuously decreases during the development of the instability.

The critical Reynolds number $\left(R e_{\delta c r}\right)$ depends largely on the position of the point 

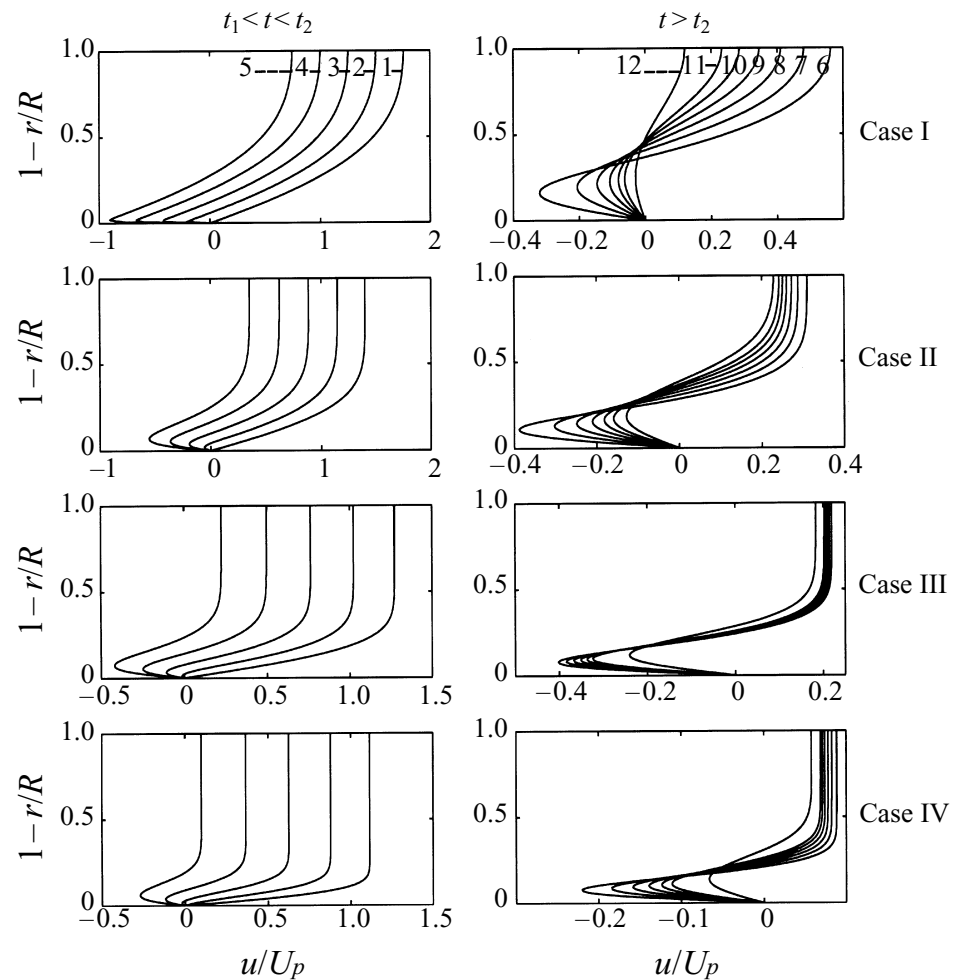

FiguRE 10. Velocity profiles at different times after the motor is switched off for the four cases; curve $1, t=t_{2} ; 2, t=t_{1}+\left(t_{2}-t_{1}\right) / 4 ; 3, t=t_{1}+2\left(t_{2}-t_{1}\right) / 4 ; 4, t=t_{1}+3\left(t_{2}-t_{1}\right) / 4 ; 5, t=t_{2}$; $6, t=t_{2}+\left(t_{p}-t_{2}\right) / 6 ; 7, t=t_{2}+2\left(t_{p}-t_{2}\right) / 6 ; 8, t=t_{2}+3\left(t_{p}-t_{2}\right) / 6 ; 9, t=t_{2}+4\left(t_{p}-t_{2}\right) / 6 ; 10$, $t=t_{2}+5\left(t_{p}-t_{2}\right) / 6 ; 11, t=t_{p} ;$ and $12, t=t_{v}$.

of inflection $\left(\delta_{i}\right)$ (Das, Arakari \& Vashist 1995). After the motor is switched off the point of inflection forms and moves away from the wall (figure 11) and the profiles tends to become more unstable. However, the reduction of the Reynolds number with time makes the profiles more stable. Whether the instability continues to grow depends on the balance between these two competing influences. Also the reduction of $\Delta u$ with time implies decreasing of growth rates of the disturbances.

\subsubsection{Linear stability}

The critical Reynolds numbers are from a linear stability analysis of the velocity profiles shown in figure 10. We have assumed plane flow with antisymmetric perturbations across the centreline. (Of course it would have been more appropriate to assume an axisymmetric geometry with helical perturbations. We believe, however, the results would not be very different.) We consider the disturbance stream function of the form of a Tollmien-Schlichting wave, $\phi=\phi(y) \mathrm{e}^{\mathrm{i} \alpha(x-c t)}$ where $\alpha$ is the wavenumber and $c=c_{r}+\mathrm{i} c_{i}, c_{r}$ is the phase speed and $c_{i}$ is the growth rate. The Orr-Sommerfeld equation is solved for the velocity profile at each time during the decaying stage of the flow. The equation is discretized using the central difference technique and is solved from the centreline to the wall. The eigenvalues are obtained using the QZ algorithm available in MATLAB. The code was validated by reproducing the eigen spectrum of Mack (1976) for plane Poiseuille flow, and the results of Hall \& Parker (1976).

The growth rates at different times after the motor is switched off corresponding 

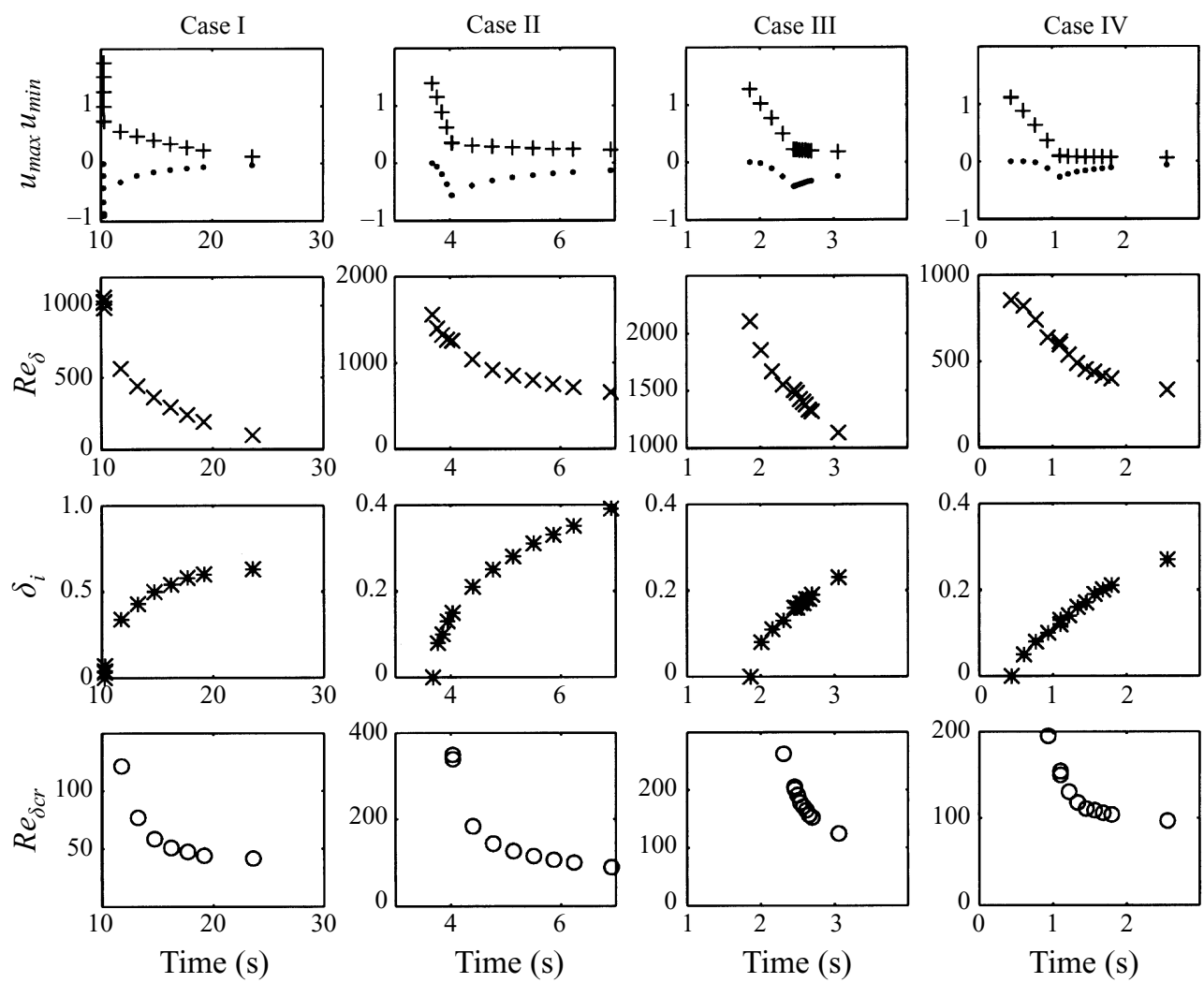

FIGURE 11. Calculated normalized maximum velocity, minimum velocity, local Reynolds number, point of inflection and critical Reynolds number as a function of time after the motor is switched off for the four cases.

to the four cases are shown in figure 12(a). The striking feature of this figure is that the wavenumbers corresponding to maximum growth rate do not change appreciably with time for each of the four cases. A similiar observation was made by Gad-el-Hak et al. (1984) in their linearly decelerated plate experiment. Note, however, that the $\alpha$ corresponding to maximum growth rate normalized by $\delta_{1}$ are different for the four cases. The growth rates initially increase as the velocity profiles become more unstable and then decrease as the velocity difference $(\Delta u)$ reduces. $R e_{\delta c r}$ rapidly decreases and does not change appreciably during the development of the instability. The values of Reynolds numbers are high enough that the instability is essentially inviscid. The effect of viscosity is mainly to reduce $\Delta u$ and thus the growth rate. This reduction of growth rate is the main reason for the non-breakdown of the vortices in case I and IV even though the Reynolds numbers in both cases were well above the critical.

We next look at the stability characteristics non-dimensionalized by the conditions at each instant of time. This is motivated by the analysis by Das et al. (1995) who concluded that for stability of inflectional velocity profiles with reverse flow near a wall

(a) the proper velocity scale is $\Delta u=u_{\max }-u_{\min }$ and the proper length scale is the boundary layer thickness, and

(b) the critical Reynolds number increases as the inflection point moves closer to the wall. 

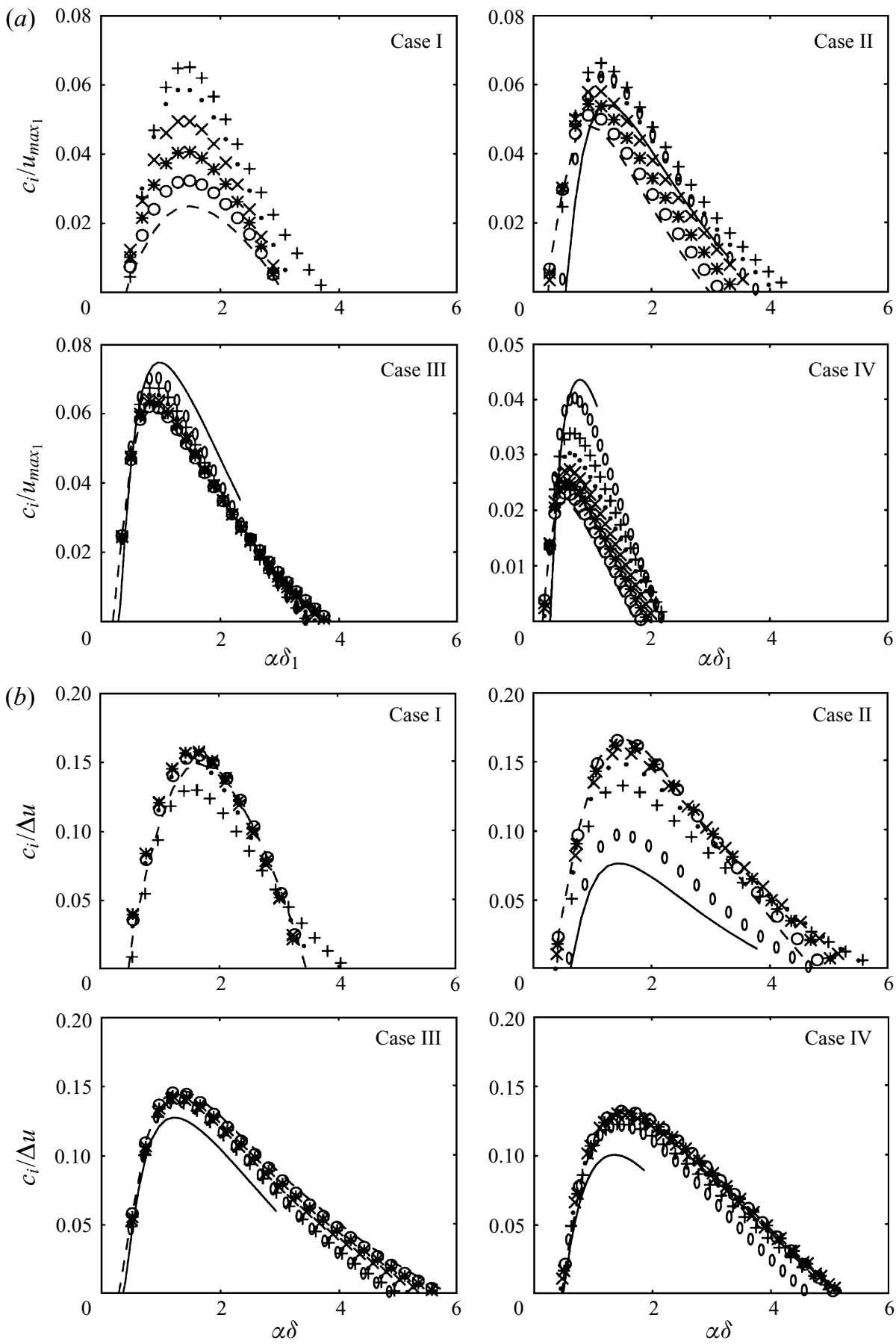

FigURE 12. (a) Growth rate as function of wavenumber at different times for the four cases; growth rate $\left(c_{i}\right)$ is non-dimensionalized by centreline velocity at $t_{1}\left(u_{\max 1}\right)$ and wavenumber $\alpha$ is non-dimensionalized with boundary layer thickness at $t_{1}\left(\delta_{1}\right)$. solid line, $t=t_{1}+3\left(t_{2}-t_{1}\right) / 4 ; O$, $t=t_{2}+\left(t_{p}-t_{2}\right) / 6 ;+, t=t_{2} ; \mathrm{o}, t=t_{2}+2\left(t_{p}-t_{2}\right) / 6 ; \cdot, t=t_{2}+3\left(t_{p}-t_{2}\right) / 6 ;{ }^{*}, t=t_{2}+4\left(t_{p}-t_{2}\right) / 6$; $\times, t=t_{2}+5\left(t_{p}-t_{2}\right) / 6$; dashed line, $t=t_{p}$. (b) As $(a)$ but growth rate $\left(c_{i}\right)$ is non-dimensionalized by local velocity difference $(\Delta u)$ and wavenumber $\alpha$ is non-dimensionalized with local boundary layer thickness $(\delta)$. 
It was shown that the neutral stability curves collapse when non-dimensionalized as above for a range of reverse flow velocities but fixed inflection point position. Growth rates normalized by the instantaneous $\Delta u$ versus wavenumber normalized by the instantaneous boundary layer thickness are shown in figure 12(b). There is some dependence of growth rate on inflection point position, but most of the curves show a maximum growth rates of about $0.15 \Delta u$, a value typical for shear layer instability, at $\alpha \delta \simeq 1.5$. This conclusion - collapse of stability characteristics when scaled with the instantaneous variables $\Delta u$ and $\delta$ - helps us to scale the experimentally obtained values of wavelength of the instability and time to instability.

\subsection{Wavelength and time of formation of the instability}

We have experimentally observed that instability results in vortex formation and its breakdown makes the flow turbulent. Keeping in mind that our aim is to understand the evolution of the reverse flow type velocity profiles, we measure the times when the wave appears, when the vortex forms, and when transition occurs and the wavelength of the wave appears.

Dimensional analysis can reveal the length and time scales to non-dimensionalize the above measures of instability. In all the experiments we observe instability after the piston has stopped. Thus we assume the parameters which govern the instability are $u_{\text {max }}, \delta_{1}, v, R$ and the deceleration time $\left(t_{2}-t_{1}\right)$. From these we get the following non-dimensional parameters:

$$
\frac{u_{\max } \delta_{1}}{v}, \quad \frac{\delta_{1}}{R}, \quad \frac{\left(t_{2}-t_{1}\right) u_{\max }}{\delta_{1}} .
$$

The first two are respectively equivalent to the Reynolds number, $R e_{\delta_{s t}}$, and the Stokes parameter $\Lambda$ in the oscillating pipe flow. The last parameter is a non-dimensional deceleration time; for an impulsively stopped piston case this parameter drops out. From dimensional analysis thus we can write the following relations for the times to instability, vortex formation and transition and the wavelength of the instability:

$\frac{\left(t_{p}-t_{1}\right) u_{\max _{1}}}{\delta_{1}}, \quad \frac{\left(t_{v}-t_{1}\right) u_{\max _{1}}}{\delta_{1}}, \quad \frac{\left(t_{t r}-t_{1}\right) u_{\max }}{\delta_{1}}, \quad \frac{\lambda}{\delta_{1}} \sim f\left(\frac{u_{\max _{1}} \delta_{1}}{v}, \frac{\delta_{1}}{R}, \frac{\left(t_{2}-t_{1}\right) u_{\max _{1}}}{\delta_{1}}\right)$.

Note that $u_{\max }$ and $\delta_{1}$ will depend on the piston velocity history prior to the start of the deceleration phase, i.e. on $U_{p}, t_{0},\left(t_{1}-t_{0}\right)$. In our experiments there was not much control over the time $\left(t_{2}-t_{1}\right)$. Hence a systematic study of the effect of the deceleration parameter cannot be made. However, we are able to get a few experiments where $\delta_{1} / R$ and $\left(t_{2}-t_{1}\right) u_{\max _{1}} / \delta_{1}$ are nearly constant. For these cases, the variation of $\left(t_{p}-t_{1}\right) u_{\max } / \delta_{1}$ with $R e_{\delta_{1}}=u_{\max _{1}} \delta_{1} / v$ are shown in figure 13 . It may be observed that more rapid the deceleration shorter is the (non-dimensional) time to instability. As expected, one can observe in this figure that the non-dimensional time for instability is independent of $R e_{\delta_{1}}$ when both the parameters $\delta_{1} / R$ and $\left(t_{2}-t_{1}\right) u_{\max _{1}} / \delta_{1}$ are kept constant.

There is another way, which we follow, to non-dimensionalize the results. This allows us to collapse the data from all the experiments. Linear stability analysis of the previous section suggests $\delta$ and $\delta / \Delta u$ as appropriate scales. These quantities, however, vary with time in each of the experiments; thus, we take time-averaged values. Another relevant conclusion from the stability analysis is that the wavenumber corresponding to maximum growth rate does not change much with time. Since the instability is essentially inviscid the disturbance will grow on the convective time scale $\delta / \Delta u$. Then 


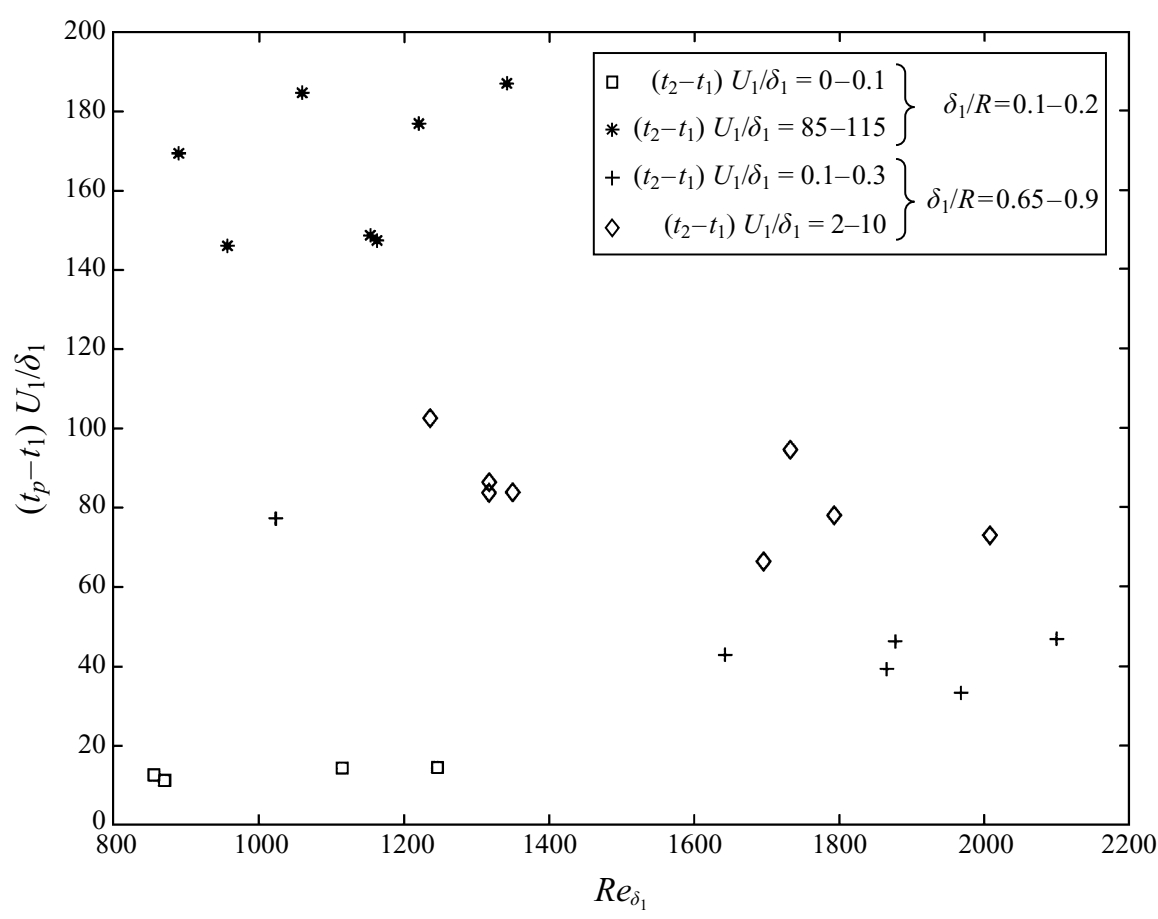

FIGURE 13. Effect of the different non-dimensional parameters on the time to instability.

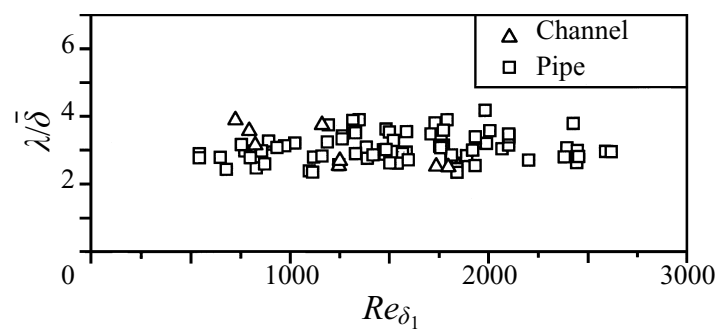

FIGURE 14. Variation of experimentally measured value of distance between adjacent vortices $(\lambda)$ non-dimensionalized by average (average taken over $t_{1}$ and $t_{p}$ ) boundary layer thickness $(\bar{\delta})$ with different $R e_{\delta_{1}}$.

we may expect the time to wave formation to scale like $1 / \overline{(\Delta u / \delta)}$, the average taken between $t_{1}$ and $t_{p}, t_{v}$ and $t_{t r}$.

The wavelengths non-dimensionalized by the average boundary layer thickness $\bar{\delta}$, average taken over the time $t_{1}$ to $t_{p}$, are shown in figure 14 . The average value of all the points of figure 14 is 3.0 with standard deviation of 0.4 , i.e.

$$
\frac{\lambda}{\bar{\delta}}=3
$$

There is some scatter which is to be expected considering that the different experiments have widely different velocity histories. We might get a better collapse if averaging is done by weighting with the growth rate at each time.

In table 2 we compare the experimentally obtained wavelength, $\lambda$, with $\lambda_{\text {crit }}$ ( $\lambda$ corresponding to $R e_{\delta c r}$ ) and $\lambda_{c i \max }$ ( $\lambda$ corresponding to maximum growth rate). (Both 


$\begin{array}{lccccc}\text { Case } & \lambda / \bar{\delta} & \lambda_{\text {crit }} / \bar{\delta} & \lambda_{\text {ci } \max } / \bar{\delta} & \int^{t_{p}} \alpha c_{i} \mathrm{~d} t & \int^{t_{v}} \alpha c_{i} \mathrm{~d} t \\ \text { I } & 3.2 & 3.3 & 4.0 & 5.8 & 6.5 \\ \text { II } & 2.7 & 3.4 & 4.2 & 6.6 & 7.6 \\ \text { III } & 3.1 & 3.4 & 5.0 & 4.5 & 6.3 \\ \text { IV } & 3.0 & 2.8 & 3.3 & 5.1 & 5.8\end{array}$

TABLE 2. Comparison of wavelengths.
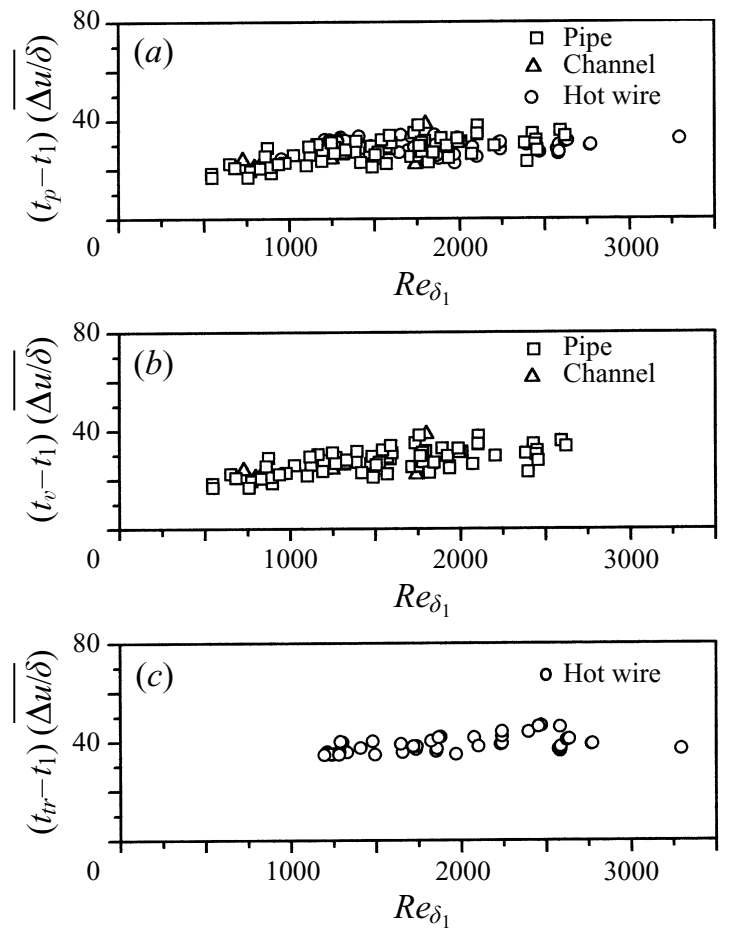

FIGURE 15. (a) Variation of experimentally measured time when the dye line becomes wavy and the time when the flow ceases to be unidirectional obtained from the hot-wire traces after the motor is switched off. Time is non-dimensionalized by average convective time scale $1 /(\overline{\Delta u / \delta})$ (average taken over $t_{1}$ and $t_{p}$ or $\left.t_{p h}\right)$. (b) Variation of experimentally measured time when vortices are formed after the motor is switched off. Time is non-dimensionalized by average convective time scale $1 /(\overline{\Delta u / \delta})$ (average taken over $t_{1}$ and $t_{v}$ ). (c) Variation of experimentally measured transition time after the motor is switched off. Time is non-dimensionalized by average convective time scale $1 /(\overline{\Delta u / \delta})$ (average taken over $t_{1}$ and $t_{t r}$ ).

$\lambda_{\text {crit }}$ and $\lambda_{\text {cimax }}$ change with time but not by much. Table 2 gives the average values.) Except for case IV, $\lambda_{\text {cimax }}>\lambda$. We do not know the reason for the small discrepancy. One possibility is that the stability analysis is for plane flow whereas the experiments are in a circular cross-section geometry. Note in case IV (for which $\lambda \simeq \lambda_{\text {ci } \max }$ ) the boundary layer thickness is small compared to the pipe radius and thus curvature effects are expected to be negligible. However, the experimentally observed wavelength is close to $\lambda_{\text {crit }}$ in all the four cases. The values of $\lambda_{\text {ci max }} / \bar{\delta}$ for the four cases lie between 3.3 and 5.0 (table 2), and indicates that the scatter in figure 14 is due more to real differences than to experimental uncertainties. 
The time $\left(t_{p}\right)$ when the wave appears obtained from flow visualization and the time $\left(t_{p h}\right)$ when the flow ceases to be unidirectional obtained from the hot-wire traces are shown in figure $15(a)$. Note that there is no systematic difference between $t_{p}$ and $t_{p h}$. There is some $R e_{\delta_{1}}$ dependence. For $R e_{\delta_{1}}>1000$

$$
\left(t_{p}-t_{1}\right)\left(\frac{\overline{\Delta u}}{\delta}\right) \simeq 29 .
$$

For $R e_{\delta_{1}}<500$ we have not observed any wave formation. Figure 15(a) may be compared with figure 13 where the other normalization is used.

The non-dimensional vortex formation time shown in figure $15(b)$ shows more scatter with

$$
\left(t_{v}-t_{1}\right)\left(\frac{\overline{\Delta u}}{\delta}\right) \simeq 33 .
$$

The non-dimensional transition time (figure $15 c$ ) shows slight increase with Reynolds number. Its average value is given by

$$
\left(t_{b}-t_{1}\right)\left(\frac{\overline{\Delta u}}{\delta}\right) \simeq 39 .
$$

Note that transition to turbulence is observed only for $\operatorname{Re}_{\delta_{1}}>1200$.

The scatter in the data can be partly attributed again to trying to find a single time scale for the experiments which have such widely different velocity histories and partly to the difficulty in precisely defining a time for the formation of the wave and the vortex. Such scatter in flow visualization data and transition data is not uncommon (Thorpe 1971). The non-dimensional transition time, which is determined from hot-wire traces and thus more precisely defined, shows less scatter. In any case, these results are useful to estimate the time of the formation of the vortices in general unsteady flows having velocity profiles with inflection points.

The accumulated amplification of a disturbance with wavenumber $\alpha$ till time $t$ is given by $\exp \int_{0}^{t} \alpha c_{i} \mathrm{~d} t$. Table 2 shows the calculated $\int \alpha c_{i} \mathrm{~d} t$ values for wave formation and for vortex formation in each of the four cases. The average (for the four cases) amplification for the wave formation is $\mathrm{e}^{5.5}$ and for vortex formation is $\mathrm{e}^{6.5}$. These values may be compared to the amplification of $\mathrm{e}^{9}$ used to predict-transition point in steady wall bounded flows (see White 1991).

\subsection{Oscillatory pipe flow}

Many of the results pertaining to oscillating pipe flow are summarized in Akhavan et al. $(1991 a, b)$. Reynolds number, $R e_{\delta_{s t}}$ (based on amplitude of velocity, $U_{0}$ and Stokes layer thickness $\delta_{s t}=((2 v) / \omega)^{1 / 2}$, where $\omega$ is the frequency of oscillation) and $\Lambda$ (ratio of radius of the pipe and $\delta_{s t}$ ) are two parameters which describe these flows. In this two-parameter space oscillatory pipe flow can be divided as first shown by Hino et al. (1976) into four regimes: (I) laminar flow, (II) disturbed laminar flow, (III) intermittently turbulent flow and (IV) fully turbulent flow. In the flows of type (II) deviation from the laminar flow by a small amplitude occurs during the acceleration phase of the cycle. In type (III), flow remains laminar in the acceleration phase but turbulent bursts are detected during the deceleration phase of the cycle. For high enough $R e_{\delta_{s t}}$ flow may become turbulent during the acceleration phase itself; see below. The transitional Reynolds number, $R e_{\delta_{s t} c r}$, for type III flow reported by different investigators (Sergeev 1966; Hino et al. 1976; Ohmi et al. 1982; Spalart \& 
Baldwin 1987; Jensen et al. 1989; Akhavan et al. 1991a,b; Eckmann \& Grotberg 1991 ) is between 500 to 550 and is independent of $\Lambda$ for $\Lambda>2$. In the case of flow over an oscillating plate transitional $R e_{\delta_{s t} c r}$ is reported as 565 (Li 1954). Merkli \& Thomann (1975) have observed $R e_{\delta_{s t} c r} \approx 280$ probably for flows of type II. Hino et al. (1976) and Ohmi et al. (1982) have observed similar $R e_{\delta_{s t} c r}$ value for type II flow.

\subsubsection{Explanation of the four regimes}

It is possible to explain some observations of stability and transition in the zero mean oscillatory pipe flow using the results of our experiments. The two key results from our experiments are that for the vortices to appear the Reynolds number should be above the critical value and there should be enough time for the instability to grow. If either of these conditions is not satisfied laminar flow (type I flow) will be observed. If there is enough time for the vortices to form then the disturbed laminar flow (type II flow) will be observed, and if the vortices break down intermittent turbulent flow (type III) will be observed.

Consider type II (disturbed laminar) flow first. We expect the instability (of the inflectional velocity profiles) to be initiated during the deceleration phase. It is crucial to realize this fact. In figure 16 the calculated velocity profiles for $\Lambda=10$ and for piston velocity $u_{p}(t)=U_{0} \sin (\omega t)$ are shown at eight phases for half a cycle starting from the start of the deceleration phase. The inflection points together with the expected instability vortex direction are also shown. We are looking at the same 'mode' (clockwise) which develops during the deceleration phase and continues into the acceleration phase. Note that at the start of the deceleration phase this is not the most unstable mode; the most unstable mode (anticlockwise), which however would rapidly decay, is that associated with the inflection point further away from the wall. For $\omega t>6 \pi / 8$ (phase 2) inflectional velocity profiles with reverse flow appear. The neutral stability curves corresponding to the different phases are shown in figure 17. The most unstable profile appears at $\omega t=\pi$ with a critical $R e_{\delta_{s t}}=82$. Flow with $R e_{\delta_{s t}}>82$ may be expected to be unstable. But experimental instability in the form of disturbed laminar flow is obtained at $R e_{\delta_{s t}} \simeq 280$.

The growth rates for the different phases for $R e_{\delta_{s t}} \approx 300$ are shown in figure 18 . As for the velocity profiles in our experiments we observe that the wavenumber corresponding to the maximum value of $c_{i}$ does not change significantly with time. Positive growth rates $\left(c_{i}>0\right)$ are obtained approximately between phases $2(\omega t=$ $6 \pi / 8)$ and $7(\omega t=11 \pi / 8)$; the instability is initiated in the deceleration phase and continues into the acceleration phase. This is why the 'disturbed laminar flow' (i.e. after the perturbations have grown sufficiently) is observed in the acceleration phase of the cycle. For any Reynolds number less than 300 there is insufficient time for the disturbance to grow and for the vortices to form. Type II flow possibly is similar to our case I and case IV flows (figure 3 and figure 7 respectively) where vortices form but do not break down.

As the Reynolds number is increased the instability starts growing from earlier in the deceleration phase (Hino et al. 1976; Jensen et al. 1989) and the flow may become turbulent by breakdown of the vortices and we have type III flow. This appears to happen at $R e_{\delta_{s t}} \simeq 550$. For $R e_{\delta_{s t}} \simeq 550$ from figure 17 we see that the flow becomes unstable starting from phase $1(\omega t=5 \pi / 8)$.

Clearly once the flow becomes turbulent the velocity profile at the beginning of the next deceleration phase at $\omega t=3 \pi / 2$ will be affected. If the flow becomes turbulent in the deceleration phase $(\omega t<\pi)$ itself then the mean velocity profile at the beginning of the acceleration phase, because of turbulent mixing, may be expected to be zero. In 

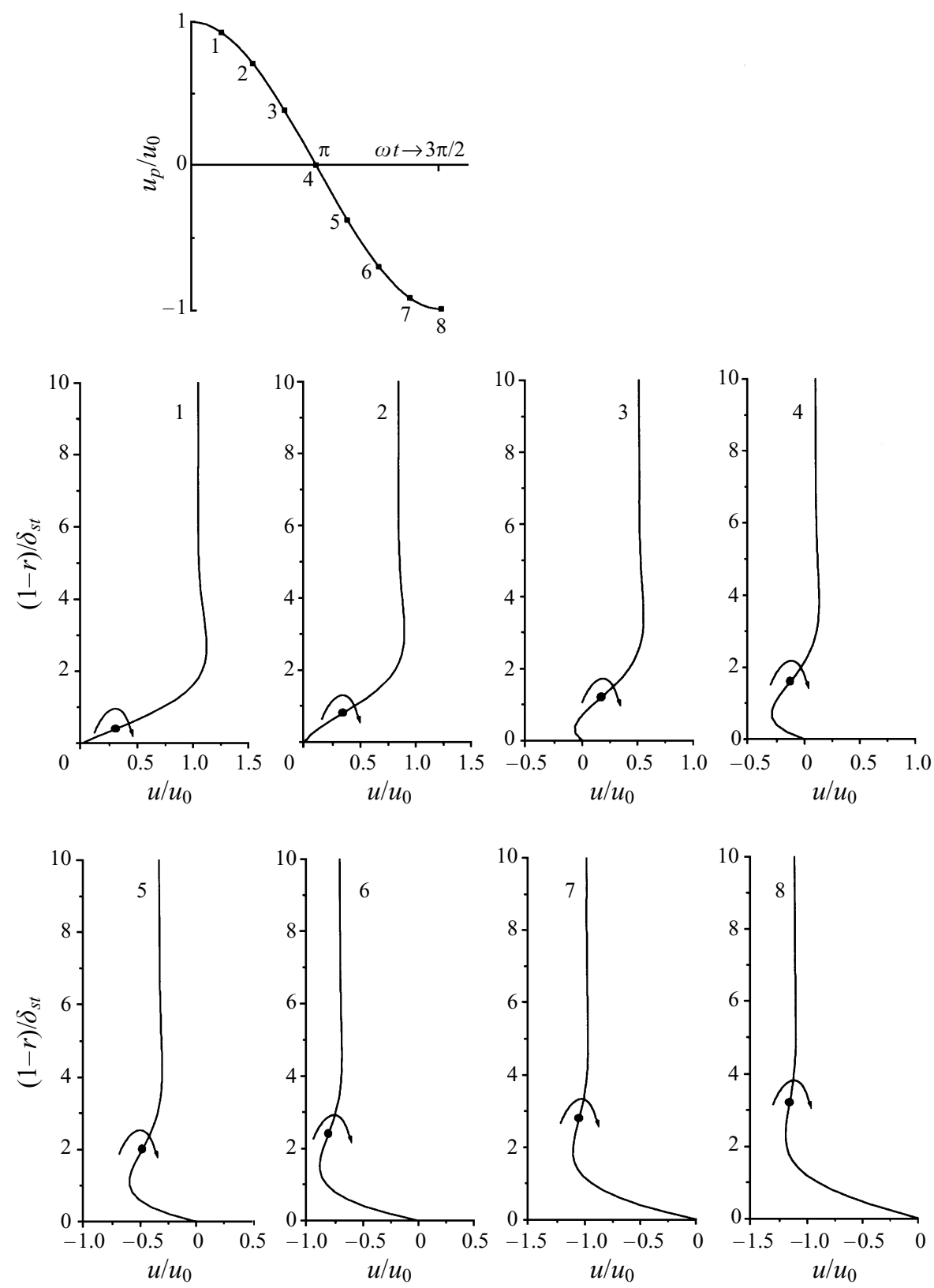

FIGURE 16. Velocity profiles for $\Lambda=10$ in oscillating pipe flow. The point of inflection, velocity scale $(\Delta u)$, boundary layer thickness $(\delta)$ and the expected instability vortex direction are shown. (1) $\omega t=5 \pi / 8$, (2) $\omega t=6 \pi / 8$, (3) $\omega t=7 \pi / 8$, (4) $\omega t=\pi$, (5) $\omega t=9 \pi / 8,(6) \omega t=10 \pi / 8$, (7) $\omega t=11 \pi / 8$, (8) $\omega t=3 \pi / 2$.

this case the flow and instability will be similar to what is obtained in our experiments, i.e. flow accelerated starting at $\omega t=0$ from zero velocity across the pipe cross-section and deceleration starting from $\omega t=\pi / 2$. In our experiments deceleration is rapid and transition is observed after the piston has stopped but in the oscillating pipe flow deceleration is gradual and transition is observed during the deceleration phase itself. 


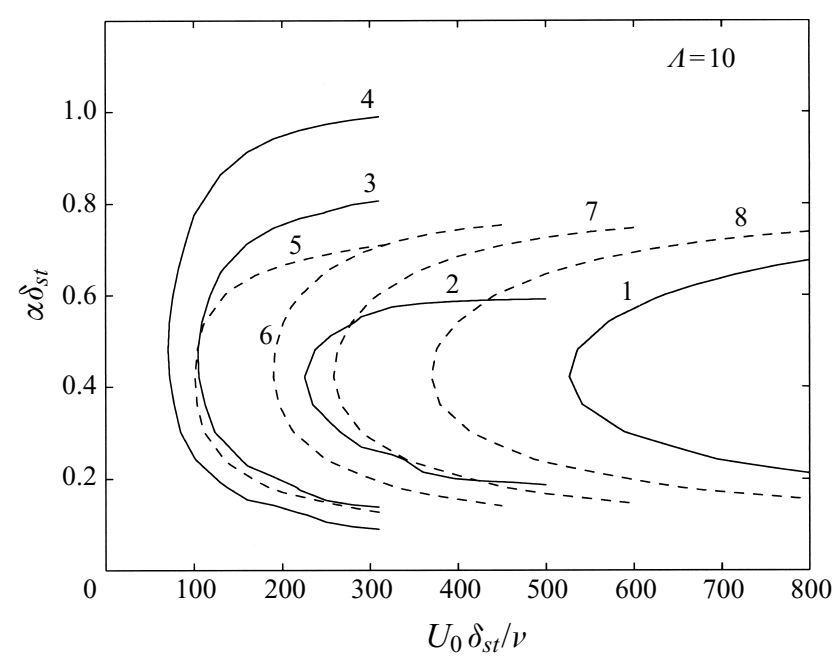

FIGURE 17. Neutral stability curves for different phases of oscillating pipe flow: (1) to (8) correspond to the same $\omega t$ as in figure 16.

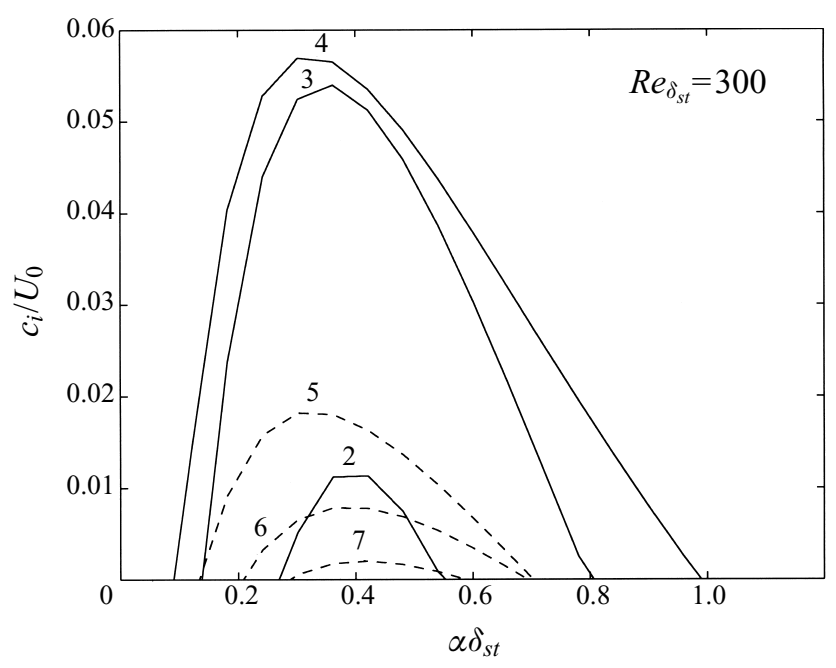

FIGURE 18. Growth rates for different phases for $R e_{\delta_{s t}} \simeq 300$ of oscillating pipe flow:

(1) to (8) correspond to the same $\omega \mathrm{t}$ as in figure 16.

Another crucial difference is that unlike in our experiments the oscillating pipe flow contains background residual turbulent fluctuations.

If the Reynolds number is further increased then the flow can be unstable, and become turbulent, during the acceleration phase itself. This will be instability of the non-inflectional velocity profiles. For example, experiments of Akhavan et al. (1991a) at $R e_{\delta_{s t}}=1080$, corresponds to this situation. Thus we can really break up the type III flow into two categories: one where the transition occurs during the deceleration phase and the second where the transition occurs during the acceleration phase. In experiments on transition to turbulence in a constant-acceleration pipe flow Lefebvre $\&$ White (1989) observe the transition process of non-inflectional velocity profiles. In their case also transition time scales with the convective time scale. As the Reynolds 


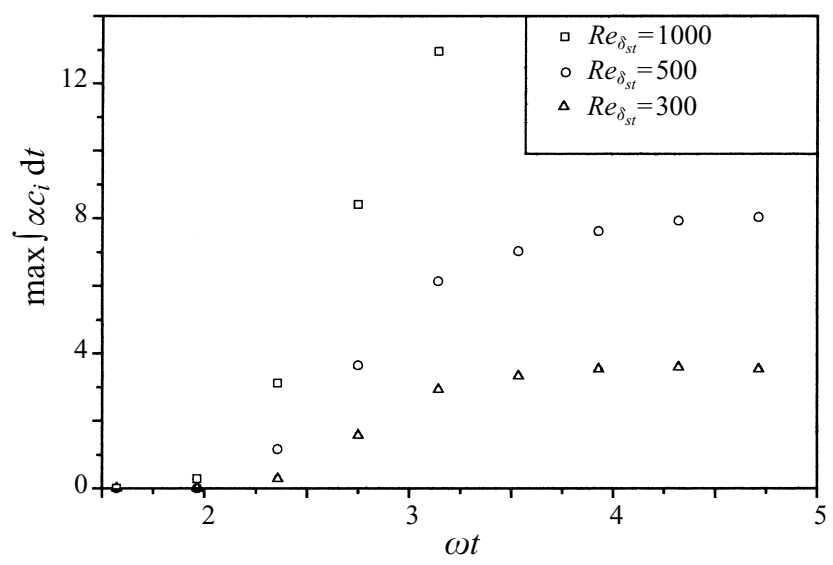

FIGURE 19. Variation of $\max \left(\int \alpha c_{i} \mathrm{~d} t\right)$ vs. $\omega t$ for $\Lambda=10$ and Reynolds number of 300,500 and 1000.

\begin{tabular}{|c|c|c|c|c|}
\hline$R e_{\delta_{s t}}$ & $\Lambda$ & $\begin{array}{c}\omega t \text { when } \\
\int \alpha c_{i} \mathrm{~d} t=4\end{array}$ & $\begin{array}{l}\omega t \text { when } \\
\int \alpha c_{i} \mathrm{~d} t=5\end{array}$ & $\begin{array}{l}\omega t \text { transition from } \\
\text { experiments (Ref.) }\end{array}$ \\
\hline 180 & 3.9 & $1.3 \pi$ & Does not reach 5 & $1.1 \pi$ (Hino et al. 1976$)$ \\
\hline 500 & 3.9 & $0.84 \pi$ & $0.88 \pi$ & $0.78 \pi$ (Hino et al. 1976) \\
\hline 543 & 1.91 & $0.82 \pi$ & $0.85 \pi$ & $0.7 \pi$ (Hino et al. 1976$)$ \\
\hline 1000 & 10 & $0.77 \pi$ & $0.79 \pi$ & $0.38 \pi$ (Akhavan et al. $(1991 a)$ \\
\hline 1530 & 1.91 & $0.66 \pi$ & $0.68 \pi$ & $0.45 \pi$ (Hino et al. 1976$)$ \\
\hline
\end{tabular}

TABLE 3. Comparison of phase values.

number is increased even further the transition point will occur earlier and earlier in the acceleration phase and eventually at very high Reynolds numbers type IV (fully turbulent) flow may be observed.

\subsubsection{Prediction of transition}

If we assume transition occurs when the growth (from the linear stability analysis) $\int \alpha c_{i} \mathrm{~d} t$ reaches a certain critical value we can predict the phase at which transition occurs for a given Reynolds number. In our experiments we observe wave formation when $\int \alpha c_{i} \mathrm{~d} t \sim 5$ and vortex formation when $\int \alpha c_{i} \mathrm{~d} t \sim 6$. Turbulence occurs, in those cases where it does, shortly after vortex formation.

Figure 19 shows the growths at $\Lambda=10$ for three Reynolds numbers, $\operatorname{Re}_{\delta_{s t}}=300$, 500 and 1000. (The velocity profiles used for $R e_{\delta_{s t}}=300$ and 500 and in table 3 for $R e_{\delta_{s t}}=180$ and 500 are for oscillating laminar flow; for $R e_{\delta_{s t}}=1000$ and in table 3 for $R e_{\delta_{s t}}=543,1000$ and 1530 the velocity profiles are calculated assuming the velocity $=0$ across the cross section at $\omega \mathrm{t}=0$.)

From figure 19 and the experimentally observed $R e_{\delta_{s t} c r} \simeq 300$ for type II flow we see that for oscillating pipe flow $\int^{t_{p}} \alpha c_{i} \mathrm{~d} t \simeq 4$. Possibly, a higher disturbance level in the oscillating flow causes the $\int \alpha c_{i} \mathrm{~d} t$ value to be lower than in our experiments. Further, we may assume that transition occurs when $\int \alpha c_{i} \mathrm{~d} t \simeq 5$. Figure 19 predicts the experimental finding that instability is observed 'earlier' as the Reynolds number is increased. $\int \alpha c_{i} \mathrm{~d} t \simeq 4$ at $\omega \mathrm{t}=0.96 \pi, 0.84 \pi$ and $0.77 \pi$ for $R e_{\delta_{s t}}=300,500$ and 1000 respectively. Table 3 compares the phase values when $\int \alpha c_{i} \mathrm{~d} t=4,5$ with 
the phase values at which transition is observed in experiments. The predictions and experiments reasonably agree at the lower Reynolds numbers.

However, at the higher Reynolds numbers (1000 and 1530) the agreement is poor: the observed transition is in the acceleration phase while the prediction is in the deceleration phase. While the mean velocity profile (till transition) is well predicted by assuming laminar flow (Akhavan et. al. 1991a) the linear stability analysis is unable to predict the phase at which transition occurs. Clearly the background turbulence causes transition to occur much earlier; in fact, before the flow becomes linearly unstable. Lefebvre \& White (1989) in their linearly accelerated pipe flow experiments obtain a transitional Reynolds number based on boundary layer thickness $\simeq 25000$. The effect of background turbulence on the transition in unsteady flows needs more study. (A vast literature exists on the effect of turbulence on transition in steady flows (Schlichting 1979)). A simple way would be an oscillating flow experiment at high enough $R e_{\delta_{s t}}$, say 1500 , to record the phases at which transition occurs starting from the first cycle. Initially the background turbulence will be very low and then it will build up to the asymptotic level in a few cycles. The changes in the transition phase with each cycle will reveal the effect of background turbulence.

\subsubsection{Transition time}

A general result can be derived for the transition time. We have $t_{i}$, the time to instability or transition, scaling with the convective time scale $t_{i} \sim \delta / U_{0}$. Further $\delta \sim$ $\delta_{s t}=(v \tau / \pi)^{1 / 2}$, where $\tau$ is the time period of oscillation. Thus using $\operatorname{Re}_{s s t}=U_{0} \delta_{s t} / v$, we have $t_{i} / \tau \sim \delta v / U_{0} \delta_{s t}^{2} \sim 1 / R e_{\delta s t}$. Thus as the Reynolds number is increased the transition time in relation to the time period will reduce.

\subsubsection{Independence of critical Reynolds number of $\Lambda$}

From the literature it is seen that in oscillating pipe flow the critical Reynolds numbers (based on $\delta_{s t}$ ) for disturbed laminar flow and transition to intermittent turbulent flow are independent of $\Lambda$ for $\Lambda>2$, i.e. for $\delta_{s t}<R / 2$. The important parameters for determining instability and transition are the position of the point of inflection, $\delta_{i}$, and the convective time scale, $\delta / \Delta u$. The independence of $\Lambda$ of the critical Reynolds numbers is understood from figure 20 $(a, b)$ which shows that $\delta_{i} / \delta_{s t}$ and $\left(\delta_{s t} / U_{0}\right) /(\delta / \Delta u)$ plotted versus $\omega t$ tend to become independent of $\Lambda$ for $\Lambda>2$. Here $\Delta u$ is the velocity difference corresponding to the shear layer at each phase (see figure 16).

\subsection{Unsteady boundary layer separation}

During unsteady boundary layer separation velocity profiles with reverse flow are obtained prior to formation of vortices and the eventual breakaway of the layer close to the wall. In some cases the formation of the vortices appears because of the instability of the reverse flow profiles. The main difficulty in extending the results of our experiments to unsteady separation is the spatial variations of the pressure gradient and of the external velocity usually obtained in the latter. It can be shown that the effect of the spatial variation is likely to be less at high enough values of Reynolds number because then the boundary layer thickness will be small compared to the scale of the spatial variation. Then, the results from $\S 5.4$ (equation (5.3)) can be used to estimate the time to formation of the vortices.

There is a strong Reynolds number dependence of the process of formation and the initial size of the separation region. At low Reynolds numbers separation vortices form scaling with the diameter. At high Reynolds numbers multiple vortices form 
Transition of unsteady velocity profiles with reverse flow
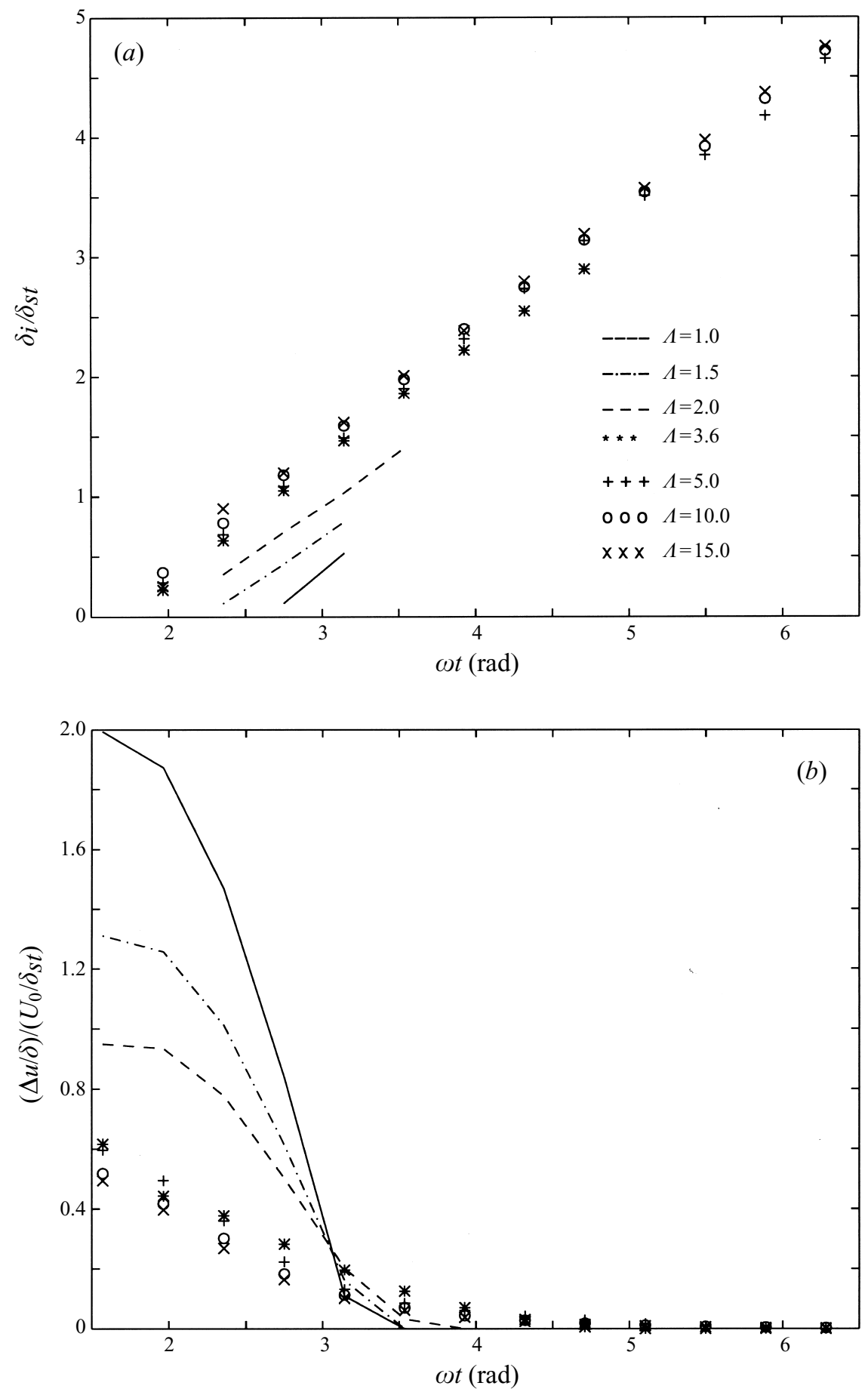

FigURE 20. Variation of $(a) \delta_{i} / \delta_{s t}$ and $(b)(\Delta u / \delta) /\left(U_{0} / \delta_{s t}\right)$ with $\omega t$ for different values of $\Lambda$. 
scaling with the boundary layer thickness. At the highest $R e_{D}, 9500$, for an impulsively started cylinder for which experiments have been done (Bouard \& Coutanceau 1980) the first vortex forms at $t U / D \simeq 1.0$ at an angle of $140^{\circ}$ from the upstream stagnation point. Using relation (5.3) we estimate the time to vortex formation to be $t U / D \simeq 1.1$ (Das 1998). In the dynamic stall experiments at high Re (see McAlister \& Carr 1979) the so-called shear layer vortices are observed. These are believed to be the result of instability of the reverse flow region. A rough calculation in this case also shows that the time to vortex formation calculated using equation (5.3) tallies with experimental observations. The flow subsequent to the initial vortex formation will of course be governed by vortex dynamics. In any case onset of dynamic stall may be estimated by using relation (5.3).

The view we are taking of unsteady boundary layer separation - instability of the boundary layer - is different from that of Van Dommelen \& Shen (1982) which is that separation is related to singularity in the boundary layer solution. For an impulsively started cylinder they observe singularity after $0.75 \mathrm{D}$ movement at $\theta=$ $111^{\circ}$. These values appear to be independent of $R e_{D}$ in contradiction to experimental observations.

\section{Conclusion}

The simple trapezoidal piston motion allows us to study the instability and transition of unsteady inflectional point profiles. Even at large Reynolds numbers viscous effects are important in that the velocity profiles change significantly during the instability. However, the instability is essentially inviscid and the growth rates scale with the convective velocities. Whether the flow becomes unstable or not depends on whether there is enough time for the instability to grow. The time to instability is approximately $29 / \overline{\Delta u / \delta}$, the transition time is $\sim 39 / \overline{\Delta u / \delta}$ and the wavelength of the instability is $\sim 3 \bar{\delta}$. At low Reynolds numbers $\left(R e_{\delta_{1}}<500\right)$ instability is not observed and transition to turbulence is observed only for $R e_{\delta_{1}} \geqslant 1200$. We may emphasize here that these values of the Reynolds numbers will be applicable only to flows which are rapidly decelerated. For flow in the pipe the helical mode is most unstable at least for $\delta / R$ not small compared to 1 . Similarly in channel flow the antisymmetric mode of disturbances is the more unstable for $\delta / H$ not small compared to 1 . If the $R e_{\delta}$ is high enough breakdown to turbulence is observed. At high speeds secondary vortices are induced at the wall.

Linear stability analysis gives an important result that the wavenumber corresponding to maximum growth rate does not change much during the instability. Stability characteristics of reverse flow profiles for a given inflection point position collapse when scaled with $\Delta u$ (maximum velocity - minimum velocity) and boundary layer thickness $(\delta)$.

Using the results of our experiments and quasi-steady linear stability analysis we can explain many observations of instability and transition in oscillating pipe flow. In unsteady boundary layer separation, also, at high $R e$, formation of the first vortex may be due to boundary layer instability.

We acknowledge the help of Mr Ashish Misra who obtained preliminary results as part of his ME project work. We thank Dr P. N. Shankar of National Aeronautical Laboratory and Professor V. H. Arakeri for going through the manuscript and suggesting changes. We thank the reviewers for their comments which greatly helped in clarifying some issues and improved the quality of the paper. 
Appendix A. Velocity calculation for given piston motion: pipe flow

In the literature no solution exists for unsteady laminar flow in a pipe or channel caused by any given but arbitrary piston motion. For sinusoidal piston motion, when the pressure also varies sinusoidally in time, a solution exists. The governing equations are (4.1), (4.3), (4.4) and (4.5) and are repeated below for convenience. The equation of motion in the $x$-direction is

$$
\frac{\partial u}{\partial t}=-\frac{1}{\rho} \frac{\partial p}{\partial x}+v\left(\frac{\partial^{2} u}{\partial r^{2}}+\frac{1}{r} \frac{\partial u}{\partial r}\right)
$$

with boundary conditions

$$
u(R, t)=0, \quad \frac{\partial u(0, t)}{\partial r}=0
$$

and initial condition

$$
u(r, o)=0 .
$$

The volume flux due to piston motion is

$$
\int_{0}^{R} 2 \pi r u \mathrm{~d} r=u_{p}(t) \pi R^{2}
$$

Taking the Laplace transform of equations (A1)-(A4) we obtain the solution in terms of the transformed variable $s$ as

$$
\bar{u}(r, s)=\frac{\overline{u_{p}}(s)\left[I_{0}\left(B s^{1 / 2}\right)-I_{0}\left(A s^{1 / 2}\right)\right]}{\left[I_{0}\left(B s^{1 / 2}\right)-\frac{2 I_{1}\left(B s^{1 / 2}\right)}{B s^{1 / 2}}\right]},
$$

where, $I_{0}$ and $I_{1}$ are modified Bessel's function of zeroth and first order respectively. $B=R / v^{1 / 2}, A=r / v^{1 / 2}$ and $\overline{u_{p}}(s)$ is the Laplace transform of piston velocity, $u_{p}$. The details of the derivation can be obtained from Das \& Arakeri (1996). The velocity profiles given in figures $1(b)$ and 10 were obtained using this method.

The inverse transform of equation (A 5) is calculated using the contour integration method as it can be shown that the convolution theorem is not applicable. Putting $B s^{1 / 2}=\mathrm{i} v$ the denominator becomes $J_{2}(v)$ where $\mathrm{i}=\sqrt{-1}$ and $J_{2}(v)$ is a Bessel function of the second kind. The poles are the zeros of $J_{2}(v)$ and $s=0$. The residues at all the poles are found to get the solution. The solution in non-dimensional form at different times for a trapezoidal piston motion shown in figure $1(b)$ are:

during piston acceleration $0 \leqslant t \leqslant t_{0}$

$$
\begin{aligned}
\frac{u}{U_{p}}=\frac{1}{t_{0}}\left[2 t\left(1-c^{2}\right)+\frac{B^{2}}{2}\right. & \left.\left(\frac{1}{4}\left(1-c^{4}\right)-\frac{1}{3}\left(1-c^{2}\right)\right)\right] \\
& +\frac{2 B^{2}}{t_{0}} \sum_{n=1}^{\infty} \exp \left(-\frac{v_{n}^{2}}{B^{2}}\right) t\left[\frac{J_{0}\left(v_{n}\right)-J_{0}\left(c v_{n}\right)}{v_{n}^{3} J_{1}\left(v_{n}\right)}\right] ;
\end{aligned}
$$

when piston velocity is constant $=U_{p}\left(t_{0} \leqslant t \leqslant t_{1}\right)$

$$
\begin{aligned}
\frac{u}{U_{p}}= & 2\left(1-c^{2}\right) \\
& +\frac{2 B^{2}}{t_{0}} \sum_{n=1}^{\infty}\left(\exp \left(-\frac{v_{n}^{2}}{B^{2}} t\right)-\exp \left(-\frac{v_{n}^{2}}{B^{2}}\left(t-t_{0}\right)\right)\right)\left[\frac{J_{0}\left(v_{n}\right)-J_{0}\left(c v_{n}\right)}{v_{n}^{3} J_{1}\left(v_{n}\right)}\right] ;
\end{aligned}
$$


during piston deceleration $\left(t_{1} \leqslant t \leqslant t_{2}\right)$

$$
\begin{aligned}
\frac{u}{U_{p}}= & 2 \frac{\left(t_{2}-t\right)}{\left(t_{2}-t_{1}\right)}\left(1-c^{2}\right)-\frac{B^{2}}{2\left(t_{2}-t_{1}\right)}\left(\frac{1}{4}\left(1-c^{4}\right)-\frac{1}{3}\left(1-c^{2}\right)\right) \\
& +2 B^{2} \sum_{n=1}^{\infty}\left(\frac{\exp \left(-\frac{v_{n}^{2}}{B^{2}} t\right)-\exp \left(-\frac{v_{n}^{2}}{B^{2}}\left(t-t_{0}\right)\right)}{t_{0}}-\frac{\exp \left(-\frac{v_{n}^{2}}{B^{2}}\left(t-t_{1}\right)\right)}{t_{2}-t_{1}}\right) \\
& \times\left[\frac{J_{0}\left(v_{n}\right)-J_{0}\left(c v_{n}\right)}{v_{n}^{3} J_{1}\left(v_{n}\right)}\right]
\end{aligned}
$$

after the piston has stopped $\left(t_{2} \leqslant t \leqslant \infty\right)$

$$
\begin{aligned}
\frac{u}{U_{p}}= & 2 B^{2} \sum_{n=1}^{\infty}\left(\frac{\exp \left(-\frac{v_{n}^{2}}{B^{2}} t\right)-\exp \left(-\frac{v_{n}^{2}}{B^{2}}\left(t-t_{0}\right)\right)}{t_{0}}\right. \\
& \left.+\frac{\exp \left(-\frac{v_{n}^{2}}{B^{2}}\left(t-t_{2}\right)\right)-\exp \left(-\frac{v_{n}^{2}}{B^{2}}\left(t-t_{1}\right)\right)}{t_{2}-t_{1}}\right)\left[\frac{J_{0}\left(v_{n}\right)-J_{0}\left(c v_{n}\right)}{v_{n}^{3} J_{1}\left(v_{n}\right)}\right] ;
\end{aligned}
$$

where $c=r / R, v_{n}$ are zeros of $J_{2}(v), B=R / v^{1 / 2}, A=R / v^{1 / 2}$ and $U_{p}=$ piston velocity during time $t_{0}$ to $t_{1}$. Note in (A7) if the piston is moved with constant velocity for long time (i.e. $t_{1} \rightarrow \infty$ ) we get the parabolic velocity profile corresponding to fully developed pipe flow.

Weinbaum \& Parker (1975) gave an approximate solution to a fully developed flow in a circular pipe and channel which is impulsively blocked. The exact solution obtained using the above method to this problem is, for pipe flow,

$$
\frac{u}{U_{0}}=2 \sum_{n=1}^{\infty} \exp \left(-\frac{v_{n}^{2}}{B^{2}} t\right)\left[\frac{J_{0}\left(v_{n}\right)-J_{0}\left(c v_{n}\right)}{v_{n} J_{1}\left(v_{n}\right)}\right]
$$

and for channel flow,

$$
\frac{u}{U_{0}}=2 \sum_{n H=1}^{\infty} \exp \left(-\frac{v_{n H}^{2}}{B^{2}} t\right)\left[\frac{\cos \left(c_{H} v_{n H}\right)-\cos \left(v_{n H}\right)}{v_{n H} \sin \left(v_{n H}\right)}\right] .
$$

Here, $U_{0}$ is the average velocity of the fully developed flow, subscript $H$ indicates the quantities for the channel, $v_{n H}$ are zeros of $\tan (v)$ and $c_{H}=y / H$. $H$ is channel half-width and $y$ is distance from centreline towards the wall of the channel.

\section{Appendix B. Velocity calculation for given piston motion: channel flow}

In the case of flow in a channel similar equations are solved and the solution at different times are: 
at $0 \leqslant t \leqslant t_{0}$

$$
\begin{aligned}
\frac{u}{U_{p}}=\frac{1}{t_{0}}\left(\frac{3 t}{2}\left(1-c_{H}^{2}\right)\right. & \left.-\frac{B_{H}^{2}}{40}\left(5 c_{H}^{4}-6 c_{H}^{2}+1\right)\right) \\
& +\frac{2 B_{H}^{2}}{t_{0}} \sum_{n H=1}^{\infty} \exp \left(-\frac{v_{n H}^{2}}{B_{H}^{2}} t\right)\left[\frac{\cos \left(c_{H} v_{n H}\right)-\cos \left(v_{n H}\right)}{v_{n H}^{3} \sin \left(v_{n H}\right)}\right] ;
\end{aligned}
$$

at $t_{0} \leqslant t \leqslant t_{1}$

$$
\begin{aligned}
\frac{u}{U_{p}}= & \frac{3}{2}\left(1-c_{H}^{2}\right) \\
& +\frac{2 B_{H}^{2}}{t_{0}} \sum_{n H=1}^{\infty}\left(\exp \left(-\frac{v_{n H}^{2}}{B_{H}^{2}} t\right)-\exp \left(-\frac{v_{n H}^{2}}{B_{H}^{2}}\left(t-t_{0}\right)\right)\right)\left[\frac{\cos \left(c_{H} v_{n H}\right)-\cos \left(v_{n H}\right)}{v_{n H}^{3} \sin \left(v_{n H}\right)}\right] ;
\end{aligned}
$$

at $t_{1} \leqslant t \leqslant t_{2}$

$$
\begin{aligned}
\frac{u}{U_{p}}= & \frac{3}{2}\left(1-c_{H}^{2}\right) \frac{\left(t_{2}-t\right)}{\left(t_{2}-t_{1}\right)}+\frac{1}{\left(t_{2}-t_{1}\right)} \frac{B_{H}^{2}}{40}\left(5 c_{H}^{4}-6 c_{H}^{2}+1\right) \\
& +2 B_{H}^{2} \sum_{n H=1}^{\infty}\left(\frac{\exp \left(-\frac{v_{n H}^{2}}{B_{H}^{2}} t\right)-\exp \left(-\frac{v_{n H}^{2}}{B_{H}^{2}}\left(t-t_{0}\right)\right)}{t_{0}}-\frac{\exp \left(-\frac{v_{n H}^{2}}{B_{H}^{2}}\left(t-t_{1}\right)\right)}{t_{2}-t_{1}}\right) \\
& \times\left[\frac{\cos \left(c_{H} v_{n H}\right)-\cos \left(v_{n H}\right)}{v_{n H}^{3} \sin \left(v_{n H}\right)}\right] ;
\end{aligned}
$$

at $t_{2} \leqslant t \leqslant \infty$

$$
\begin{aligned}
\frac{u}{U_{p}}= & 2 B_{H}^{2} \sum_{n H=1}^{\infty}\left(\frac{\exp \left(-\frac{v_{n H}^{2}}{B_{H}^{2}} t\right)-\exp \left(-\frac{v_{n H}^{2}}{B_{H}^{2}}\left(t-t_{0}\right)\right)}{t_{0}}+\right. \\
& \left.\frac{\exp \left(-\frac{v_{n H}^{2}}{B_{H}^{2}}\left(t-t_{2}\right)\right)-\exp \left(-\frac{v_{n H}^{2}}{B_{H}^{2}}\left(t-t_{1}\right)\right)}{t_{2}-t_{1}}\right)\left[\frac{\cos \left(c_{H} v_{n H}\right)-\cos \left(v_{n H}\right)}{v_{n H}^{3} \sin \left(v_{n H}\right)}\right] .
\end{aligned}
$$

\section{REFERENCES}

Akhavan, R., Kamm, R. D. \& Shapiro, A. H. 1991a An investigation of transition to turbulence in bounded oscillatory Stokes flows. Part 1. Experiments. J. Fluid Mech. 225, 395-422.

Akhavan, R., Kamm, R. D. \& Shapiro, A. H. $1991 b$ An investigation of transition to turbulence in bounded oscillatory Stokes flows. Part 2. Numerical simulations. J. Fluid Mech. 225, 423-444.

Bouard, R. \& COUtANCEAU, M. 1980 The early stage of the wake behind an impulsively started cylinder for $40<R e<10^{4}$. J. Fluid Mech. 101, 583-607.

Cassel, K. W., Smith, F. T. \& Walker, J. D. A. 1996 The onset of instability in unsteady boundary-layer separation. J. Fluid Mech. 315, 223-256.

CebecI, T. 1982 Unsteady separation. In Proc. Symp. on Numerical and Physical Aspects of Aerodynamic Flows (ed. T. Cebeci), pp. 265-277. Springer.

Collins, J. I. 1963 Inception of turbulence at the bed under periodic gravity waves. J. Geophys. Res. 18, 6007-6014. 
Cowley, J. S., Van Dommelen, L. L. \& Lam, T. S. 1990 On the use of lagrangian variables in descriptions of unsteady boundary-layer separation. Phil. Trans. R. Soc. Lond. A 333, 343-378.

DAS, D. 1998 Evolution and instability of unsteady boundary-layers with reverse flow. PhD Thesis, Dept. Mech. Engng, IISc, Bangalore, India.

Das, D. \& Arakeri, J. H. 1996 Analytical solution of rapidly blocked unsteady pipe flow. Report Dept. Mech. Engng, IISc, Bangalore, India.

Das, D., Arakeri, J. H. \& Vashist, T. K. 1995 Linear stability analysis of wall bounded velocity profiles with inflection point. In Proc. Sixth Asian Congress of Fluid Mechanics (ed. Y. T. Chew \& C. P. Tso) pp. 1266-1269. World Scientific.

Doligalski, T. L., Smith, C. R. \& Walker, J. D. A. 1994 Vortex interactions with walls. Ann. Rev. Fluid. Mech. 26, 573-616.

Doligalski, T. L. \& Walker, J. D. A. 1984 The boundary layer induced by a convected two dimensional vortex. J. Fluid Mech. 139, 1-28.

Eckmann, D. M. \& Grotberg, J. B. 1991 Experiments on transition to turbulence in oscillatory pipe flow. J. Fluid Mech. 222, 329-350.

FALES, F. N. 1955 A new laboratory technique for investigation of origin of fluid turbulence. J. Franklin Inst. 259, 491-515.

Gad-el-Hak, M., Davis, S. H., McMurray, J. T. \& OrszaG, S. A. 1984 On the stability of decelerating laminar boundary layer. J. Fluid Mech. 138, 297-323.

HALl, P. \& PARKER, K. H. 1976 The stability of the decaying flow in a suddenly blocked channel flow. J. Fluid. Mech. 75, 305-314.

Hino, M., Sawamoto, M. \& Takasu, S. 1976 Experiments on transition to turbulence in an oscillating pipe flow. J. Fluid Mech. 75, 193-207.

Jensen, B. L., Sumer, B. M. \& Fredsøe, J. 1989 Turbulent oscillatory boundary layers at high Reynolds numbers. J. Fluid Mech. 206, 265-297.

LI, H. 1954 Stability of oscillatory laminar flow along a wall. Beach Erosion Board, US Army Corps. Engrs Tech. Memo. 47.

Lefebvre, P. J. \& White, F. M. 1989 Experiments on transition to turbulence in a constantacceleration pipe flow. Trans. ASME I: J. Fluids Engng 111, 428-432.

MACK, L. M. 1976 A numerical study of the temporal eigenvalue spectrum of the Blasius boundary layer. J. Fluid Mech. 73, 497-520.

McAlister, K. W. \& CARR, L. W. 1979 Water tunnel visualizations of dynamic stall. Trans. ASME $I: J$. Fluids Engng 101, 376-380.

Merkli, P. \& Thomann, H. 1975 Transition to turbulence in oscillating pipe flow. J. Fluid Mech. 68, 567-575.

Obremski, H. J. \& Fejer, A. A. 1967 Transition in oscillating boundary layer flows. J. Fluid Mech. 29, 93-111.

Ohmi, M., Iguchi, M., Kakehachi, K. \& Masuda, T. 1982 Transition to turbulence and velocity distribution in an oscillating pipe flow. Bull. JSME 25, 365-371.

Pauley, L. L., Moin, P. \& Reynolds, W. C. 1990 The structure of two-dimensional separation. J. Fluid Mech. 220, 397-411.

Puhak, R. I., Degani, A. T. \& Walker, J. D. A. 1995 Unsteady separation and heat transfer upstream of obstacles. J. Fluid Mech. 305, 1-27.

Pullin, D. I. \& Perry, A. E. 1980 Some flow visualization experiments on the starting vortex. J. Fluid Mech. 97, 239-255.

SARPKAYA, T. 1993 Coherent structures in oscillatory boundary layers. J. Fluid Mech. 253, 105-140.

Schlichting, H. 1979 Boundary Layer Theory, 7th edn. McGraw-Hill.

Schoppa, W., Hussain, F. \& Metcalfe, R. W. 1995 A new mechanism of small-scale transition in a plane mixing layer: core dynamics of spanwise vortices. J. Fluid Mech. 298, 23-80.

SEARs, W. R. \& Telionis, D. P. 1975 Boundary-layer separation in unsteady flow. SIAM J. Appl. Maths 28, 215-235.

Seminara, G. \& Hall, P. 1975 Linear stability of slowly varying unsteady flows in a curved channel. Proc. R. Soc. Lond. A346, 279-303.

SergeEv, S. I. 1966 Fluid oscillations in pipes at moderate Reynolds numbers. Fluid Dyn. 1, 121-122.

SHEN S. F. 1961 Some considerations on the laminar stability of time-dependent basic flows. J. Aero. Sci. 28, 397-404 \& 417. 
Spalart, P. R. \& Baldwin, B. S. 1987 Direct simulation of a turbulent oscillating boundary layer. Turbulent Shear Flows 6, pp. 417-440. Springer.

Thomas, L. H. 1953 The stability of plane Poiseuille flow. Phys. Rev. 91, 780-783.

THORPE, S. A. 1971 Experiments on the instability of stratified shear flows: miscible fluids. J. Fluid Mech. 46, 299-319.

Van Dommelen, L. L. \& Shen, S. F. 1982 Genesis of separation. In Proc. Symp. on Numerical and Physical Aspects of Aerodynamic Flows, pp. 293-311. (ed. T. Cebeci). Springer.

Walker, J. D. A. 1978 The boundary layer due to rectilinear vortex. Proc. R. Soc. Lond. A359, $167-188$.

White, F. M. 1991 Viscous Fluid Flow, 2nd edn. McGraw-Hill.

Weinbaum, S. \& Parker, K. 1975 The laminar decay of suddenly blocked channel and pipe flows. J. Fluid Mech. 69, 729-752.

Yang, K. S., Spalart, P. R. \& Ferziger, J. H. 1992 Numerical studies of natural transition in a decelerating boundary layer. J. Fluid Mech. 240, 433-468. 